
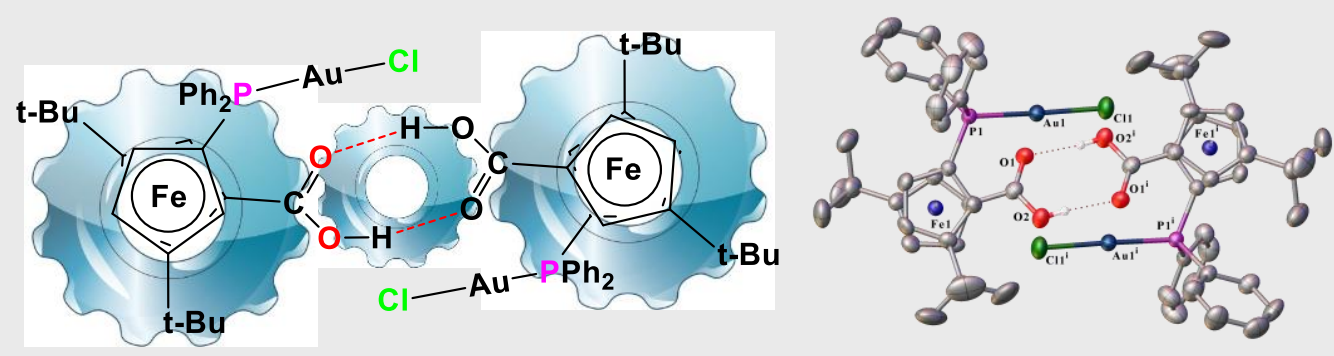

The synthesis of highly functionalized ferrocenes bearing both acidic/basic functions ( $\mathrm{PR}_{2}, \mathrm{R}=\mathrm{Ph}, \mathrm{Cy}, i-\mathrm{Pr}$, and carboxylic acids) and additional structuring substituents (bulky $t$-Bu groups) is reported along with their coordination behavior and solid-state structures.
Ambiphilic Ferrocenes*

Léa Radal, Petr Vosáhlo, Julien Roger, Hélène Cattey, Régine Amardeil, Ivana Císařová, Petr Štěpnička*, Nadine Pirio*, and Jean-Cyrille Hierso*

Highly Functionalized Brønsted Acidic/Lewis Basic Hybrid Ferrocene Ligands: Synthesis and Coordination Chemistry

TO CITE THIS PAPER:

L. Radal, P. Vosáhlo, J. Roger, H. Cattey, R. Amardeil, l. Císařová, P. Štěpnička, N. Pirio, J.-C. Hierso, Eur. J. Inorg. Chem. 2019, 865-874

DOI: 10.1002/ejic.201801378. 


\title{
Highly Functionalized Brønsted Acidic/Lewis Basic Hybrid Ferrocene Ligands: Synthesis and Coordination Chemistry
}

\author{
Léa Radal, ${ }^{[a]}$ Petr Vosáhlo, ${ }^{[b]}$ Julien Roger, ${ }^{[a]}$ Hélène Cattey, ${ }^{[a]}$ Régine Amardeil, ${ }^{[a]}$ Ivana Císařová, ${ }^{[b]}$ \\ Petr Štěpnička, ${ }^{*[b]}$ Nadine Pirio ${ }^{*[a]}$, and Jean-Cyrille Hierso*[a,c]
}

\begin{abstract}
A general challenging issue in the synthesis of hemilabile ferrocene ligands is the access to highly functionalized ferrocene starting materials. These can bear donor/acceptor atoms, additional functional and structuring spectator substituents, but the preparation of such species poses general synthetic difficulties. We report herein alternative synthetic routes to hybrid ferrocene donors such as tertbutylated phosphanylcarboxylic acids and their corresponding aldehydes. These hybrid ambiphilic species that combine Brønstedacidic and Lewis-basic functional moieties in their structure were characterized by multinuclear NMR and single-crystal X-ray diffraction analysis. In the solid state, the mutual arrangement of functional groups at the ferrocene unit is highly dependent on hydrogen bonding interactions despite the structural hindering effect of the tert-butyl groups introduced. The ligands were converted to gold $(I)$ complexes and phosphane selenides to further monitor the properties and mutual influence of the functional groups in the prepared compounds.
\end{abstract}

\section{Introduction}

The chemistry of functionalized ferrocenes evolved from the simple but widely applied chelating diphosphane, 1,1'bis(diphenylphosphanyl)ferrocene (dppf), ${ }^{[1,2]}$ to the synthesis of tailored ditopic hemilabile ferrocenyl phosphane ligands. ${ }^{[1 a, 3,4]}$ Early on, the synthesis of phosphanyl ferrocenes bearing an additional polar group $\mathrm{R}$ on the other ring of the ferrocene backbone (Scheme 1a) has been pursued by the Štěpnička group, with $\mathrm{R}$ being carboxyl group. ${ }^{[5]}$ This work was further extended to numerous carboxamides, ${ }^{[6]}$ amidosulfonates, ${ }^{[7]}$ and other compounds that can serve as hybrid ligands and/or synthetic building blocks. ${ }^{[8]}$

A general limiting aspect of the synthesis of hemilabile ferrocenes still remains an access to highly functionalized polysubstituted ferrocene derivatives as starting materials. These can bear various functional and structuring spectator substituents, and their preparation clearly involves synthetic difficulties arising mainly from the need of multiple functionalization and functional group compatibility. In this context, the Hierso group designed highly functionalized hybrid ligands and polyphosphanes (1,1',3,3-tetrafunctionalized) using dialkylated 1,1'-tertbutylferrocene as a scaffold, ${ }^{[9]}$ and cousin compounds with geminally dimethylated substituents (Scheme 1b). ${ }^{[10]}$ These compounds were used as ligands promoting metal-catalysed $C_{-}$ $\mathrm{C}$ and $\mathrm{C}-\mathrm{X}$ bond formation $\left(\mathrm{X}=\mathrm{O}, \mathrm{S}\right.$, etc.). ${ }^{[11]}$ New functionalized ferrocenes, which include both $\mathrm{P}, \mathrm{B}$ - and $\mathrm{N}, \mathrm{B}$-ambiphiles were also developed as molecular systems that combine Lewis acidic and Lewis basic groups on a common ferrocene platform. ${ }^{[2,13]} \mathrm{A}$ further functionalization at ferrocene core was demonstrated to introduce novel properties such as planar chirality and steric control over the conformation of the metallocene backbone. ${ }^{[11,13}$ 14]

By combining these approaches, we examined herein synthetic pathways to new hybrid ferrocene ligands, tert-butylated phosphanylferrocene carboxylic acids and their corresponding aldehydes, that combine Brønsted acidic and Lewis basic functional moieties in their molecules, searching for an enhanced steric control within these compounds. Different synthetic pathways were investigated and we identified the most convenient road. The ligands were converted to gold(I) complexes and to phosphane selenides to further establish the properties and mutual influence of the functional groups in the prepared compounds. All these species were characterized by multinuclear NMR and by single-crystal X-ray diffraction analysis.

[a] L. Radal, Dr. J. Roger, Dr. H. Cattey, Dr. R. Amardeil, Prof. Dr. N. Pirio, Prof. Dr. J.-C. Hierso

Institut de Chimie Moléculaire de l'Université de Bourgogne (ICMUB) UMR CNRS 6302

Université de Bourgogne Franche-Comté (UBFC)

9 avenue Alain Savary, 21078 Dijon, France

E-mail: nadine.pirio@u-bourgogne.fr; jean-cyrille.hierso@ubourgogne.fr

[b] P. Vosáhlo, Dr. I. Císařová, Prof. Dr. P. Štěpnička Department of Inorganic Chemistry

Faculty of Science, Charles University Hlavova 2030, 12840 Prague, Czech Republic E-mail: petr.stepnicka@natur.cuni.cz

[c] Prof. Dr. J.-C. Hierso

Institut Universitaire de France 103 Boulevard Saint Michel, 75005 Paris Cedex, France 


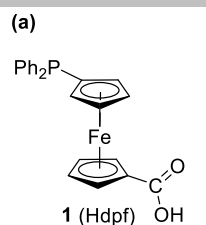

1 (Hdpf) $\mathrm{O} H$

(b)

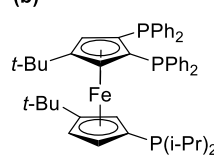

4

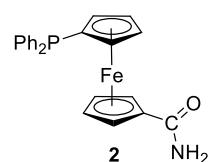

$\mathrm{Ph}_{2} \mathrm{P}$

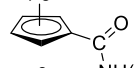

$3 \quad \mathrm{NH}\left(\mathrm{CH}_{2}\right)_{n} \mathrm{SO}_{3}\left(\mathrm{HNEt}_{3}\right)$

Stepnicka et al.

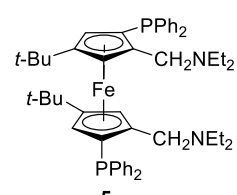

5

Hierso et al.

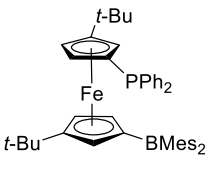

6
Scheme 1. Functionalized ferrocenylphosphane hybrid derivatives (conformations encountered in the solid state structures are depicted).

\section{Results and Discussion}

The synthesis of the target compound $\mathbf{1 0}$ (Scheme 2) has been achieved by ring opening of 1-phenyl-1-phospha[1]ferrocenophane with phenyllithium, ${ }^{[15]}$ followed by in situ carboxylation with excess solid carbon dioxide. This procedure, previously used to synthesize Hdpf (1), ${ }^{[5 a]}$ was adopted for the synthesis of 10 starting from the convenient precursor 1,1'-di-tertbutylferrocene 7 (Scheme 2). $\left.{ }^{[12,}{ }^{14}\right]$ The tert-butylated ferrocenophane 9 was characterized in $\mathrm{CDCl}_{3}$ solution by a ${ }^{31} \mathrm{P}$ NMR peak at $\delta_{p}-15.1 \mathrm{ppm}$, similar to its non-tert-butylated counterpart. ${ }^{[16]}$ Ring opening of 9 was successfully achieved with $\mathrm{PhLi}$, but it was found to be troublesome by using other nucleophiles like $t$-BuLi. Acid $\mathbf{1 0}$ was next obtained by in situ carboxylation with solid or gaseous carbon dioxide and acidic workup. Characterization of $\mathbf{1 0}$ in $\mathrm{CDCl}_{3}$ solution by multinuclear NMR data showed diagnostic peaks $\left(\delta_{\mathrm{P}}=-19.5, \delta_{\mathrm{C}}(\mathrm{COOH})=\right.$ 177.6), which are consistent with those of the non-tert-butylated analogue $\left(\delta_{\mathrm{P}}=-17.6, \delta_{\mathrm{C}}(\mathrm{COOH})=177.2\right.$ in $\left.\mathrm{CDCl}_{3}\right) .{ }^{[5 \mathrm{a}]}$

The synthesis of $\mathbf{1 0}$ via ferrocenophane opening lacks modularity, especially with respect to substituents at the phosphorus atom and, in addition, showed in our hands severe purification troubles. Because we also aimed at modifying Lewis basicity of phosphane $\mathrm{PR}_{2}$ function via changing the $\mathrm{R}$ groups, we explored the use of dilithium intermediate $\mathbf{8}^{[12]}$ to diastereoselectively generate 1,1'-dibromo-3,3'-di-tert-butylferrocene (11) ${ }^{[12]}$ (Scheme 2). Compound 11 can be further used in sequential lithiation/electrophilic trapping with a variety of chlorophosphanes $^{[5 b, 17]}$ to prepare a range of electronically distinct functional phosphanes. The phosphanylferrocene bromide intermediates were isolated in their free (12-14) or in $\mathrm{P}$ protected form as borane adducts (15-17), which are both suitable for further synthesis. This is illustrated by the preparation of free acid 10 and its borane adduct 18 and, similarly, by the preparation of the corresponding aldehydes 21 and 23. ${ }^{[18]}$ Compound 21 can be both $\mathrm{OH}$-reduced and phosphane-protected by one-pot use of $\mathrm{BH}_{3} . \mathrm{SMe}_{2}$ in excess, providing alcohol 22. In the case of acid 18, we further demonstrated that the borane protecting group can be removed by using conventional methods. ${ }^{[19]}$ Herein, removal of the $\mathrm{BH}_{3}$ moiety with 1,4-diazabicyclo[2.2.2] octane (DABCO, 2.2 equiv) in toluene $\left(80^{\circ} \mathrm{C}, 18 \mathrm{~h}\right)$ provided acid 10 in $62 \%$ yield after acidic workup and chromatographic purification. Operationally simpler heating of adduct 18 in ethanol $(10 \mathrm{~h})$ afforded the deprotected acid in a considerably better $90 \%$ yield. ${ }^{[20]}$ 

paraissant au moins une fois par an, son auteur dispose, même après avoir accordé des droits exclusifs à un éditeur, du droit de mettre à disposition gratuitement dans un format ouvert, par voie numérique, sous réserve de l'accord des éventuels coauteurs, la version finale de son manuscrit acceptée pour publication, dès lors que l'éditeur met lui-même celle-ci gratuitement à disposition par voie numérique ou, à défaut, à l'expiration d'un délai courant à compter de la date de la première publication. Ce délai est au maximum de six mois pour une publication dans le domaine des sciences, de la technique et de la médecine et de douze mois dans celui des sciences humaines et sociales.

Ferrocenophane pathway
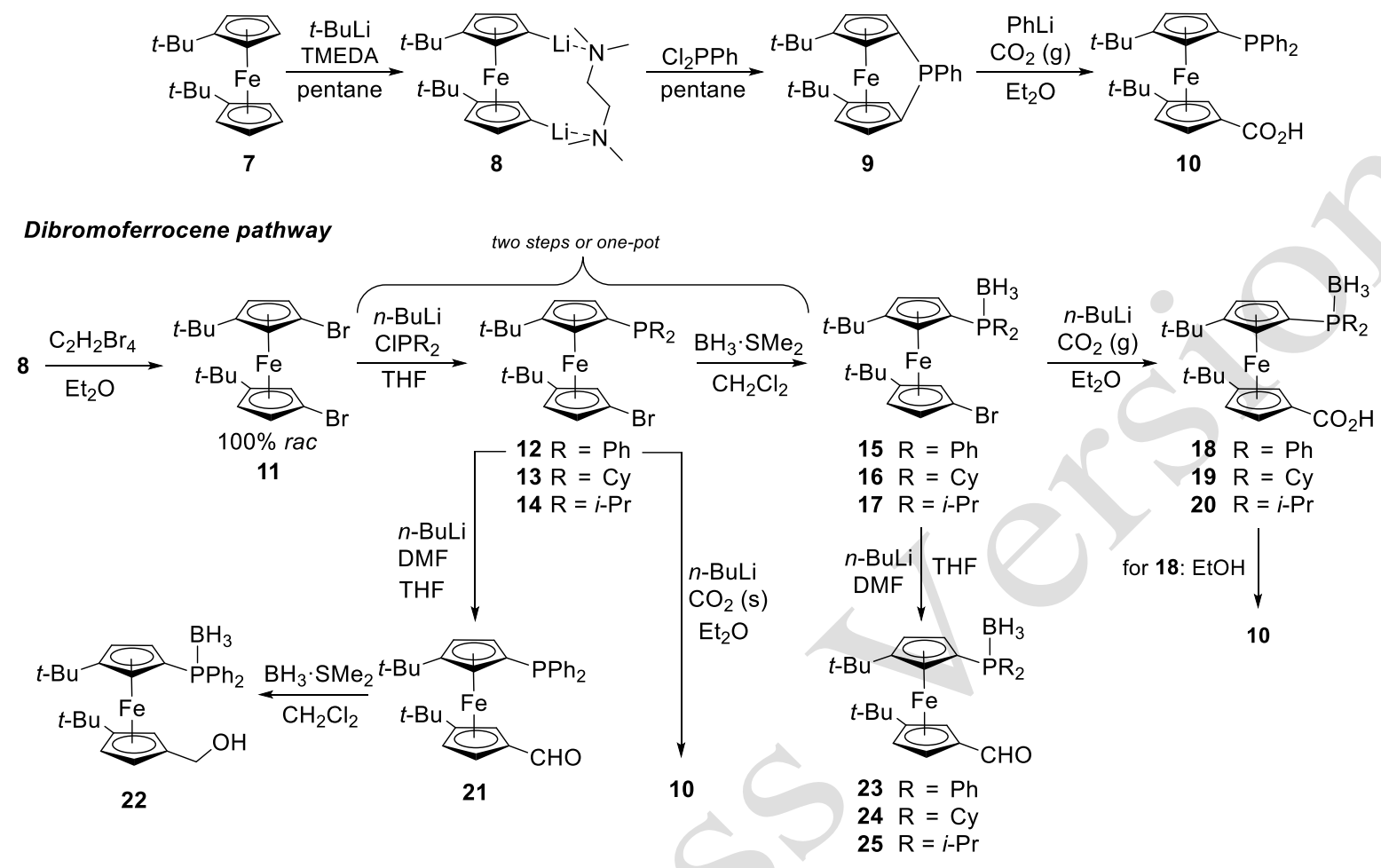

two steps or one-pot

10

Scheme 2. Synthesis of tert-butylated phosphanyl ferrocene carboxylic acids and aldehydes (TMEDA = N,N,N',N'-tetramethyl-1,2-diaminoethane). Compounds 7 , $\mathbf{8}, \mathbf{1 1}, \mathbf{1 2}, 14$ and 21 have been synthesized from reported procedures. Intermediary 13 was not isolated.

The molecular structures of 10 and $\mathbf{1 8}$ (Figure 1) were determined by single-crystal $\mathrm{X}$-ray diffraction analysis, ascertaining that both compounds are racemic, retaining the configuration of their precursor 11.

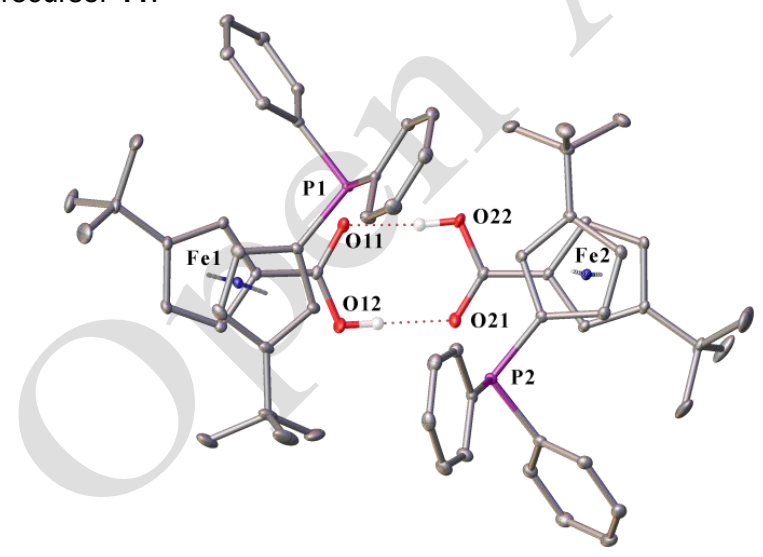

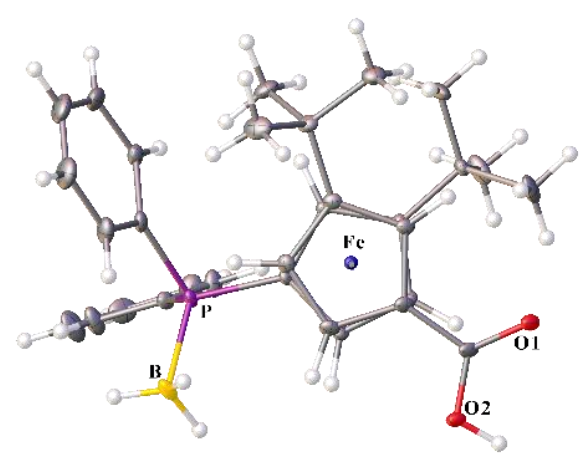

Figure 1. View of the molecular structure of $\mathbf{1 0}$ (top) and 18 (bottom). Hydrogen atoms except those in $\mathrm{OH}$ groups, are omitted for clarity. Ortep at $30 \%$ probability level.

Compound $\mathbf{1 0}$ crystallized with two structurally independent but very similar molecules, whereas in the structure of the corresponding borane adduct $\mathbf{1 8}$ there is only one structurally independent molecule. Although the orientation of the functional substituents in the molecules of $\mathbf{1 0}$ and $\mathbf{1 8}$ seems to be locked by the bulky auxiliary substituents, the ferrocene scaffolds preserves 
Lorsqu'un écrit scientifique issu d'une activité de recherche financée au moins pour moitié par des dotations de l'État, des collectivités territoriales ou des établissements publics, par des subventions d'agences de financement nationales ou par des fonds de l'Union européenne est publié dans un périodique paraissant au moins une fois par an, son auteur dispose, même après avoir accordé des droits exclusifs à un éditeur, du droit de mettre à disposition gratuitement dans un format ouvert, par voie numérique, sous réserve de l'accord des éventuels coauteurs, la version finale de son manuscrit acceptée pour publication, dès lors que l'éditeur met lui-même celle-ci gratuitement à disposition par voie numérique ou, à défaut, à l'expiration d'un délai courant à compter de la date de la première publication. Ce délai est au maximum de six mois pour une publication dans le domaine des sciences, de la technique et de la médecine et de douze mois dans celui des sciences humaines et sociales.

some of its conformational flexibility. This is shown by the torsion angles $\mathrm{P} \cdots \mathrm{Ct} 1 \cdots \mathrm{Ct} 2 \cdots \mathrm{CO}_{2} \mathrm{H}$, which are found at $52.32(3)^{\circ}$ and $53.16(3) \circ$ for 10 (two molecules) vs. 133.37(5) ${ }^{\circ}$ for $\mathbf{1 8}$, corresponding roughly to an opened 1,2-conformation and a 1,3conformation, respectively (see Tables 1 and 2). Despite the presence of bulky auxiliary substituents, the carboxyl groups remain roughly coplanar with the planes of their parent cyclopentadienyl rings (twisting $<5^{\circ}$ ). However, the ferrocene cyclopentadienyl rings are considerably more tilted in $\mathbf{1 8}$ $\left(8.73(8)^{\circ}\right)$ than in $\mathbf{1 0}\left(2.12(7)^{\circ}\right.$ and $\left.3.80(7)^{\circ}\right)$. In both structures, the individual molecules assemble into the characteristic dimers via pairs of the $\mathrm{O}-\mathrm{H} \cdots \mathrm{O}$ hydrogen bonds between the carboxyl groups from either the independent molecules $(10 ; 0 \cdots \mathrm{O}=$ 2.6363(14), 2.6279(14) $\AA$ ) or a molecule and its inversion-related counterpart (18; O ... O = 2.599(2) $\AA$ ).

We also determined the solid-state structures of the borane protected bromide 17 with $\mathrm{R}=i-\operatorname{Pr}$ (Figure 2), the boraneprotected aldehyde obtained thereof (25; Figure 3) and its analogue with cyclohexyl substituents at phosphorus atom (24; Figure 4). Selected characteristic structural parameters for these species are reported in Table 1.

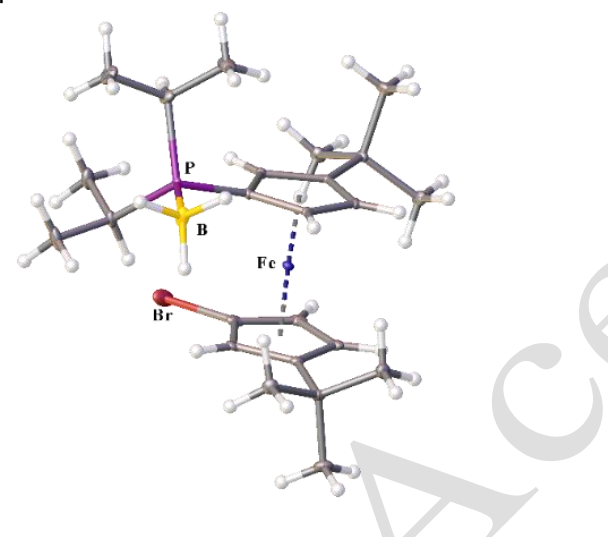

Figure 2. View of the molecular structure of $\mathbf{1 7 . 3 0 \%}$ probability level.

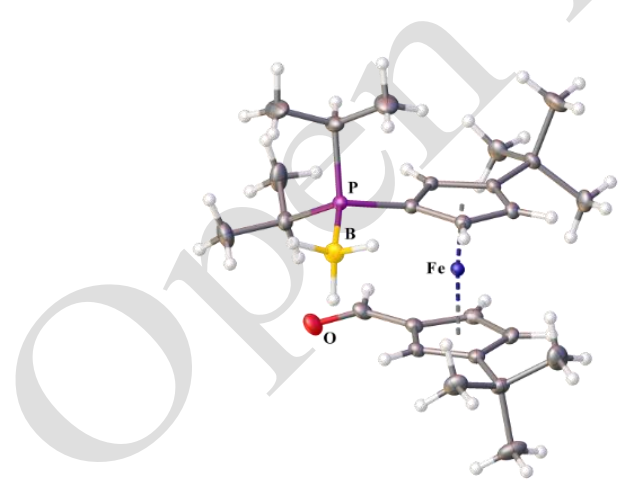

Figure 3. View of the molecular structure of $25.30 \%$ Probability level.
The general geometric features of ferrocenes 17, 24 and $\mathbf{2 5}$ are comparable. In particular, the introduced functional groups $\mathrm{Fg}$ (bromide, carboxylic acid and aldehyde, respectively) are similarly oriented with respect to the phosphane moiety with $\mathrm{P} \ldots \mathrm{Fg}$ spatial distances fairly close, ranging between approximately 4.5 and 4.9 $\AA$ in the series. The roughly opened 1,2-conformation in these compounds corresponds well to the conformation found for acid 10 and, conversely, differs from the 1,3-conformation determined for its borane adduct 18.

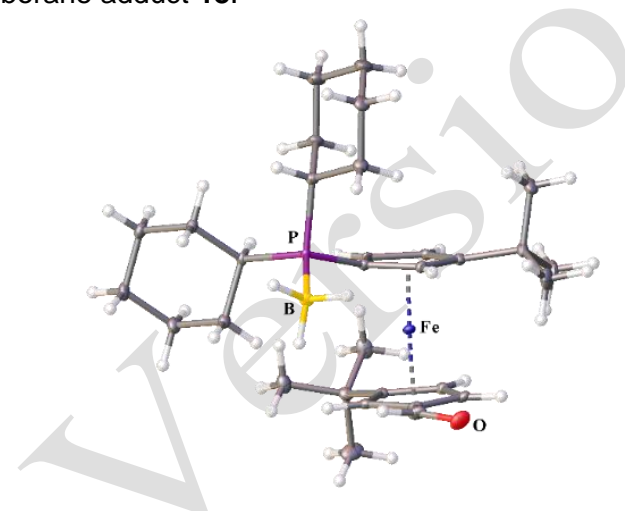

Figure 4. View of the molecular structure of 24 . $30 \%$ Probability level.

Table 1. Selected geometric parameters for 17, 24 and 25.

\begin{tabular}{|c|c|c|c|}
\hline Parameter $(\mathbf{F g})^{\mathrm{a}}$ & $17(\mathrm{Fg}=\mathrm{Br})$ & $24(\mathrm{Fg}=\mathrm{C} 27)$ & $25(\mathrm{Fg}=\mathrm{C} 21)$ \\
\hline $\mathrm{Fe}-\mathrm{Ct} 1(\AA)$ & $1.6558(7)$ & $1.6540(9)$ & $1.6615(10)$ \\
\hline $\mathrm{Fe}-\mathrm{Ct} 2(\AA)$ & $1.6579(7)$ & $1.6577(10)$ & $1.6619(10)$ \\
\hline $\mathrm{P}-\mathrm{B}(\AA)$ & $1.9153(18)$ & $1.914(2)$ & $1.917(3)$ \\
\hline $\mathrm{P} \cdots \mathrm{Fg}(\AA ̊)$ & $4.9064(5)$ & $4.621(2)$ & $4.562(2)$ \\
\hline $\mathrm{Ct} 1-\mathrm{Fe}-\mathrm{Ct} 2\left(^{\circ}\right)$ & $176.88(4)$ & $175.45(5)$ & $175.68(5)$ \\
\hline $\mathrm{P}-\mathrm{C}-\mathrm{Ct} 1\left(^{\circ}\right)$ & $170.22(12)$ & $166.00(16)$ & $170.03(16)$ \\
\hline $\mathrm{R}-\mathrm{C}-\mathrm{Ct} 2\left(^{\circ}\right)$ & $176.58(13)$ & $175.8(2)$ & $178.7(2)$ \\
\hline $\begin{array}{l}\mathrm{P} \cdots \mathrm{Ct} 1 \cdots \mathrm{Ct} 2 \cdots \mathrm{Fg} \\
\left({ }^{\circ}\right)\end{array}$ & $63.50(2)$ & $-56.42(6)$ & $56.05(6)$ \\
\hline tilt angle $\left({ }^{\circ}\right)^{[b]}$ & $2.68(6)$ & $3.91(13)$ & $3.47(12)$ \\
\hline
\end{tabular}

[a] Ct1 and Ct2 are the centroids of the phosphane- and Functional groups Fg-substituted cyclopentadienyl rings, respectively. [b] Dihedral angle of the least-squares cyclopentadienyl planes.

The electron-donating character of phosphanes is often estimated by measuring the ${ }^{1} J_{\mathrm{P}, \text { Se }}$ coupling constants of their 

établissements publics, par des subventions d'agences de financement nationales ou par des fonds de l'Union européenne est publié dans un périodique paraissant au moins une fois par an, son auteur dispose, même après avoir accordé des droits exclusifs à un éditeur, du droit de mettre à disposition gratuitement dans un format ouvert, par voie numérique, sous réserve de l'accord des éventuels coauteurs, la version finale de son manuscrit acceptée pour publication, dès lors que l'éditeur met lui-même celle-ci gratuitement à disposition par voie numérique ou, à défaut, à l'expiration d'un délai courant à compter de la date de la première publication. Ce délai est au maximum de six mois pour une publication dans le domaine des sciences, de la technique et de la médecine et de douze mois dans celui des sciences humaines et sociales.

selenide derivatives. ${ }^{[14 a]}$ An increase in these coupling constants indicates an increase in the $s$ character of the phosphorus lonepair orbital, that is, an electron-withdrawing effect of the phosphane on the selenium. We have converted acid $\mathbf{1 0}$ into the corresponding phosphane selenide 10Se (Scheme 3), which was structurally characterized (Figure 5). The ${ }^{1}$ JPSe value of $736 \mathrm{~Hz}$ determined for $\mathbf{1 0 S e}(\mathrm{d}(\mathrm{P}-\mathrm{Se})=2.1045(9) \AA)$ was identical to that of the cousin compound, 1,1'-bis(diphenylphosphanylselenoyl)$3,3^{\prime}$-di(tert-butyl)ferrocene, ${ }^{[14 a]}$ indicating that the carboxyl function exerts little influence over phosphorus in this case. In the solid state, the selenation led to an opening of the ferrocene backbone as indicated by the torsion angle $\mathrm{P} \cdots \mathrm{Ct} 1 \cdots \mathrm{Ct} 2 \cdots \mathrm{Fg}=$ $132.50(9){ }^{\circ}\left(\right.$ vs. 52.32(3) ${ }^{\circ}$ for 10), which makes the carboxyl group and the phosphane substituent more distant. Consequently, a stronger intermolecular hydrogen bonding is operating in the structure of $10 \mathrm{Se}(\mathrm{O} \cdots \mathrm{O}$ distances are shorter at 2.595(4) $\AA$ ), which is arguably due to a lower steric congestion around the carboxyl group (see Figures 1 and 5). Conversely, ferrocene cyclopentadienyl rings in $\mathbf{1 0 S e}$ are more tilted $\left(9.02(13)^{\circ}\right)$ than in the parent phosphane $10\left(2.12(7)^{\circ}\right.$ and $\left.3.80(7)^{\circ}\right)$.

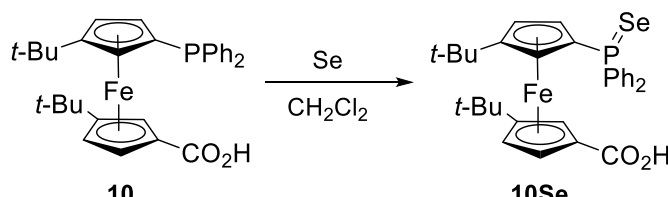

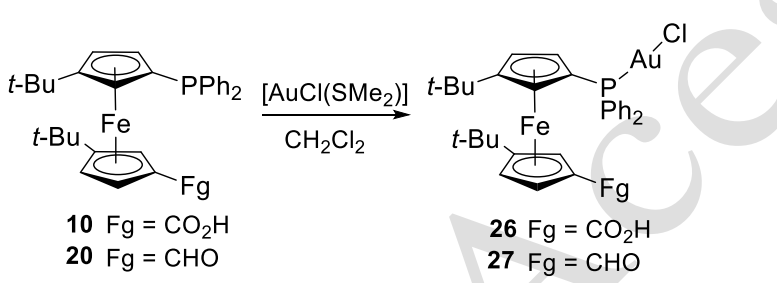

Scheme 3. Coordination chemistry of ferrocenyl hybrids to selenium or gold.

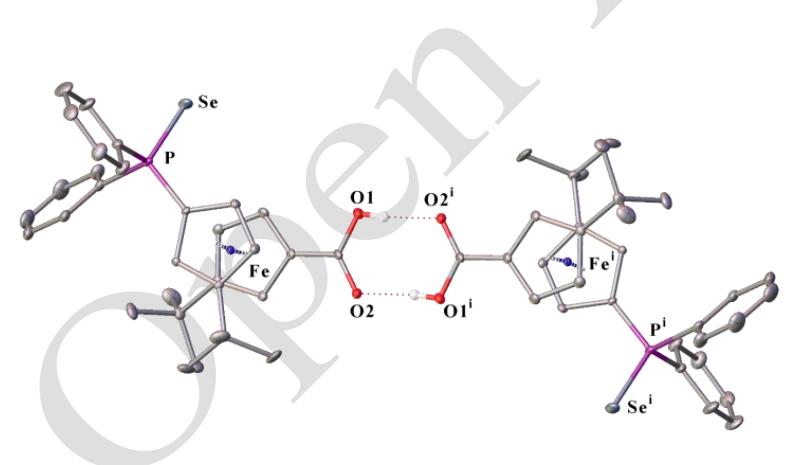

Figure 5. View of the molecular structure of 10Se (symmetry transformation: (i) $2-x, 2-y, 2-z$ ). Hydrogen atoms (except those in the $\mathrm{OH}$ groups) are omitted except those in $\mathrm{OH}$ groups. $30 \%$ Probability level.
The coordination of $\mathbf{1 0}$ to gold(I) was achieved by reacting the hybrid with $\left[\mathrm{AuCl}\left(\mathrm{SMe}_{2}\right)\right]$, leading in quantitative yield to complex 26 (Scheme 3) for which an X-ray structure analysis has also been solved (Figure 6). In the solid state, the molecules of the $\mathrm{Au}(\mathrm{I})$ complex $\mathbf{2 6}$ associate analogously to the free ligand $\mathbf{1 0}$ (cf. Fig. 1) into hydrogen bonded dimers having comparable $\mathrm{O} 1 \ldots \mathrm{O} 2 \mathrm{i}$ distances of 2.671(5) $\AA$ and O2...01i distances of 2.651(5) $\AA$. In comparison with selenation of the phosphane moiety, the coordination to gold seems to have a smaller impact on the conformation of the ambiphilic ligand, despite the higher covalent radius of gold. The gold complex presents a linear $\mathrm{P}-\mathrm{Au}-\mathrm{Cl}$ geometry (angles found to be $178.04(5)^{\circ}$ and $176.45(5)^{\circ}$ ). Interestingly, the changed conformation of the ferrocene ligand in complex $\mathbf{2 6}$ results in close proximity between the Brønsted acidic carboxylic function and the gold center with distances $\mathrm{Au} \cdots \mathrm{O}=\mathrm{COH}=3.509(4), 3.442(3) \AA$ and $\mathrm{Au} \cdots \mathrm{C}(\mathrm{O}) \mathrm{OH}=3.777(5)$, $3.720(4) \AA$, while no bonding is detected. We anticipate that this proximity might be used in assisting catalytic processes in the sphere of gold and studies into this direction are currently ongoing. Conversely, in the solid-state distances between gold centers are above $7 \AA$, precluding any aurophilic interaction. The crystal packing showed the high hindrance provided by the ferrocene ligand, which keeps the Au centers away from each.

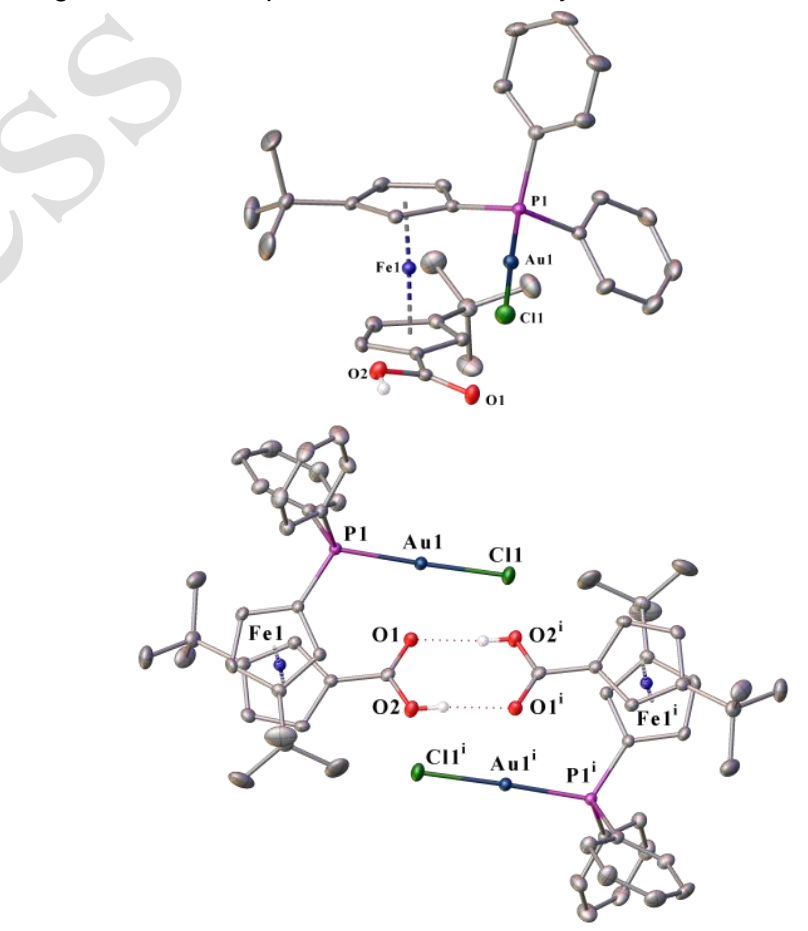

Figure 6. Views of the molecular structure of $\mathbf{2 6}$ (only one of the two molecules present in the asymmetric unit is shown here). A dichloromethane molecule and $\mathrm{H}$ atoms were omitted for clarity (except for $\mathrm{OH}$ ). Each complex formed a centrosymmetric dimer. Bonding distance P-Au: 2.2249(12) and 2.2235(11) A.. $30 \%$ Probability level. 
Lorsqu'un écrit scientifique issu d'une activité de recherche financée au moins pour moitié par des dotations de l'État, des collectivités territoriales ou des établissements publics, par des subventions d'agences de financement nationales ou par des fonds de l'Union européenne est publié dans un périodique paraissant au moins une fois par an, son auteur dispose, même après avoir accordé des droits exclusifs à un éditeur, du droit de mettre à disposition gratuitement dans un format ouvert, par voie numérique, sous réserve de l'accord des éventuels coauteurs, la version finale de son manuscrit acceptée pour publication, dès lors que l'éditeur met lui-même celle-ci gratuitement à disposition par voie numérique ou, à défaut, à l'expiration d'un délai courant à compter de la date de la première publication. Ce délai est au maximum de six mois pour une publication dans le domaine des sciences, de la technique et de la médecine et de douze mois dans celui des sciences humaines et sociales.

Table 2. Selected geometric parameters for phosphanylferrocene carboxylic acid 10 and its derivatives.

\begin{tabular}{|c|c|c|c|c|}
\hline Parameter ${ }^{[\mathrm{a}]}$ & 10 & $10 \mathrm{Se}$ & 18 & 26 \\
\hline $\mathrm{Fe}-\mathrm{Ct} 1(\AA)$ & $\begin{array}{l}1.6603(6) \\
1.6597(6)\end{array}$ & $1.6513(15)$ & $1.6711(11)$ & $\begin{array}{l}1.653(2) \\
1.664(2)\end{array}$ \\
\hline $\mathrm{Fe}-\mathrm{Ct} 2(\AA ̊)$ & $\begin{array}{l}1.6613(7) \\
1.6612(7)\end{array}$ & $1.6669(17)$ & $1.6548(10)$ & $\begin{array}{l}1.6533(19) \\
1.6563(18)\end{array}$ \\
\hline $\mathrm{P} \cdots \mathrm{CO}_{2} \mathrm{H}(\AA)$ & $\begin{array}{l}\text { P1: } \\
4.1974(13) \\
\text { P2: } \\
4.1070(13)\end{array}$ & $5.989(4)$ & $6.0188(23)$ & $\begin{array}{l}\text { P1: } \\
4.328(5) \\
\text { P2: } \\
4.317(5)\end{array}$ \\
\hline $\mathrm{O} \cdots O(\AA)^{[b]}$ & $\begin{array}{l}2.6363(14) \\
2.6279(14)\end{array}$ & $2.595(4)$ & $2.599(2)$ & $\begin{array}{l}2.671(5) \\
2.651(5)\end{array}$ \\
\hline $\mathrm{Ct} 1-\mathrm{Fe}-\mathrm{Ct} 2\left(^{\circ}\right)$ & $\begin{array}{l}177.27(4) \\
177.24(3)\end{array}$ & $175.09(8)$ & $175.25(4)$ & $\begin{array}{l}177.13(12) \\
176.52(11)\end{array}$ \\
\hline $\begin{array}{l}\mathrm{C}\left(\mathrm{CO}_{2} \mathrm{H}\right)-\mathrm{C}- \\
\mathrm{Ct} 2\left(^{\circ}\right)\end{array}$ & $\begin{array}{l}176.39(12) \\
176.96(13)\end{array}$ & $175.3(3)$ & $174.9(2)$ & $\begin{array}{l}179.2(4) \\
178.0(4)\end{array}$ \\
\hline $\begin{array}{l}\mathrm{P} \cdots \mathrm{Ct} 1 \cdots \mathrm{Ct} 2 \cdots \\
\mathrm{Fg}\left({ }^{\circ}\right)\end{array}$ & $\begin{array}{l}52.32(3) \\
53.16(3)\end{array}$ & $132.50(9)$ & $133.37(5)$ & $\begin{array}{l}52.99(13) \\
-52.90(13)\end{array}$ \\
\hline tilt angle $\left({ }^{\circ}\right)^{[c]}$ & $\begin{array}{l}2.12(7) \\
3.80(7)\end{array}$ & $9.02(13)$ & $8.73(8)$ & $\begin{array}{l}1.2(7) \\
2.4(5)\end{array}$ \\
\hline
\end{tabular}

[a] Ct1 and Ct2 are the centroids of the phosphane- and Fg-substituted cyclopentadienyl rings, respectively. [b] $\mathrm{H}$-bonding distance. [c] Dihedral angle of the least-squares cyclopentadienyl planes.

\section{Conclusions}

We report here a convenient synthetic pathway to the family of Brønsted acidic/Lewis basic ambiphilic ferrocene ligands, in particular to highly functionalized tert-butylated phosphanylferrocenecarboxylic acids bearing various phosphane

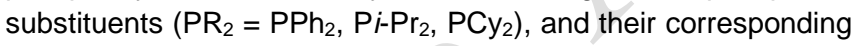
aldehydes. The role we originally attributed to the tert-butyl groups was a steric control over the conformation of the metallocene backbone, possibly enforcing a close proximity between the phosphane and carboxyl groups that could be then transferred to coordination compounds synthesized from these ligands. However, the resulting situation was more complex than we anticipated. Indeed, although the orientation of the functional substituents seems to be locked by the bulky auxiliary substituents (e.g., in the case of acid 10), the ferrocene scaffolds retains partly its conformational flexibility as it was demonstrated by its backbone conformational changes in the solid-state structures, noted upon converting phosphane 10 into borane adduct 18 or selenide $\mathbf{1 0 S e}$. Conversely, upon coordination to gold, the expected conformational control is clearly visible in the solid state (complex 26). Another noticeable point is that besides intramolecular steric interactions, the polarity of tert-butylated ferrocene carboxylic acids induce significant intermolecular hydrogen bonding, which controls the supramolecular assembly of the ferrocene derivatives in the solid state and perhaps also in solution. On-going work is focused at taking profit of these structural features exerted by the reported original ditopic ligands in cooperative transition metal catalysis.

\section{Experimental Section}

Materials and Methods. All reactions were performed under argon or nitrogen by using standard Schlenk techniques. Anhydrous THF was obtained from a Puresolv MD5 solvent drying system. Solvents used for chromatography and crystallizations were of reagent grade and were used without additional purification. NMR spectra were recorded at $25^{\circ} \mathrm{C}$ on a Varian UNITY Inova 400 (400 MHz in Prague), or ${ }^{1} \mathrm{H}$ NMR (500 and 600 $\mathrm{MHz}$ in Dijon) and ${ }^{13} \mathrm{C}$ NMR $(101,126$ or $151 \mathrm{MHz})$ spectra were recorded with a Bruker AVANCE III instrument spectrometers. Chemical shifts

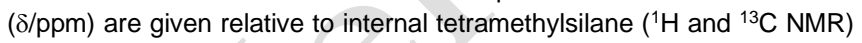
or to external, $85 \%$ aqueous $\mathrm{H}_{3} \mathrm{PO}_{4}\left({ }^{31} \mathrm{P} \mathrm{NMR}\right)$. FTIR spectra were recorded on a Nicolet 6700 spectrometer in the range $400-4000 \mathrm{~cm}^{-1}$. ESI mass spectra were obtained with a Bruker Compact Q-TOF spectrometer. Elemental analyses were performed using a Perkin-Elmer PE $2400 \mathrm{CHN}$ analyzer (in Prague), or a Thermo Electron Flash EA 1112 Series (in Dijon).

Synthesis of 10 from bromo derivative 12. Bromide 12 $^{[12]}(0.40 \mathrm{~g}, 0.71$ $\mathrm{mmol}$ ) was dissolved in dry THF $(10 \mathrm{~mL})$ and the solution was cooled in a dry ice/ethanol bath. $n$-Butyllithium $(0.49 \mathrm{~mL}$ of $1.6 \mathrm{M}$ solution, $0.78 \mathrm{mmol})$ was added and the mixture was stirred with continuous cooling for $30 \mathrm{~min}$. Next, crushed dry ice (ca. $40 \mathrm{~g}$ ) was added and the mixture was allowed to warm to room temperature (ca. $90 \mathrm{~min}$ ). Following acidification with methanolic $\mathrm{HCl}(1.1 \mathrm{~mL}$ of $1.25 \mathrm{M}, 1.4 \mathrm{mmol})$, the volatiles were removed under reduced pressure. The obtained crude product was purified column chromatography (silica gel column, dichloromethane-methanol 50:1). The first minor band was discarded and the following major one collected and evaporated to give acid $\mathbf{1 0}$ as an amorphous, red-brown solid (yield $82 \%$, $0.31 \mathrm{~g}$ ). Additional purification could be achieved through crystallization from hot hexane.

${ }^{1} \mathrm{H}$ NMR (400 MHz, $\left.\mathrm{CDCl}_{3}\right): \delta 7.60-7.55(2 \mathrm{H}, \mathrm{m}, \mathrm{Ph}), 7.39-7.34(3 \mathrm{H}$, m, Ph), $7.20-7.09$ (5 H, m, Ph), $4.73(1 \mathrm{H}, \mathrm{dd}, J=2.6,1.3 \mathrm{~Hz}, \mathrm{Cp}), 4.57$ $(1 \mathrm{H}, \mathrm{t}, J=1.5 \mathrm{~Hz}, \mathrm{Cp}), 4.29(1 \mathrm{H}, \mathrm{dd}, J=2.6,1.7 \mathrm{~Hz}, \mathrm{Cp}), 4.17(1 \mathrm{H}, \mathrm{dt}, J$ $=2.8,1.4 \mathrm{~Hz}, \mathrm{Cp}), 4.10(1 \mathrm{H}, \mathrm{dd}, J=2.5,1.5 \mathrm{~Hz}, \mathrm{Cp}), 3.90(1 \mathrm{H}, \mathrm{dt}, J=$ 2.5, 1.3 Hz, Cp), 1.26 (9 H, s, $t$-Bu), $1.04\left(9 \mathrm{H}, \mathrm{s}, t\right.$-Bu). ${ }^{13} \mathrm{C}\left\{{ }^{1} \mathrm{H}\right\} \operatorname{NMR}(101$ $\left.\mathrm{MHz}, \mathrm{CDCl}_{3}\right): \delta 177.62,140.21(\mathrm{~d}, J=12.2 \mathrm{~Hz}), 137.92(\mathrm{~d}, J=11.3 \mathrm{~Hz})$, 135.04 (d, $J=21.1 \mathrm{~Hz}), 132.35$ (d, $J=18.6 \mathrm{~Hz}), 129.08,128.28$ (d, $J=7.6$ $\mathrm{Hz}), 127.98(\mathrm{~d}, J=5.8 \mathrm{~Hz}), 127.69,106.99(\mathrm{~d}, J=5.5 \mathrm{~Hz}), 106.45,76.56$ (d, $J=10.4 \mathrm{~Hz}), 73.75$ (d, $J=26.9 \mathrm{~Hz}), 71.31,71.09,70.24,69.26,69.02$, 68.77, 31.52, $31.37(\mathrm{~d}, J=0.9 \mathrm{~Hz}), 30.86,30.51 .{ }^{31} \mathrm{P}\left\{{ }^{1} \mathrm{H}\right\} \mathrm{NMR}(162 \mathrm{MHz}$, $\mathrm{CDCl}_{3}$ ): $\delta$-19.49. IR (Nujol): $v_{\max } 3087 \mathrm{w}, 3071 \mathrm{w}, 3055 \mathrm{w}, 3041 \mathrm{w}, 2637$ w, 2598 w, 2548 w, 1668 vs, 1586 m, 1571 w, 1477 s, 1446 m, 1437 m, 1338 w, 1302 m, 1252 s, 1219 w, 1182 m, 1172 m, 1099 w, 1080 m, 1070 m, 1049 m, 1039 w, 1028 m, 998 w, 978 w, 940 w, 921 m, 902 w, 866 m, 850 m, 821 m, 783 w, 750 m, 740 s, 697 s, 680 w, 671 w, 626 w, 611 w, 558 m, 532 w, 509 m, 493 m, 466 m, 440 w cm${ }^{-1}$. Anal. Calc. for $\mathrm{C}_{31} \mathrm{H}_{35} \mathrm{FeO}_{2} \mathrm{P}$ (526.43): C 70.73, H 6.70\%. Found C 70.63\%, H 6.63\%. ESI+ MS calc. for $\mathrm{C}_{31} \mathrm{H}_{36} \mathrm{FeO}_{2} \mathrm{P}\left([\mathrm{M}+\mathrm{H}]^{+}\right): 527.1797$, found: 527.1788 .

Synthesis of 10 via ferrocenophane $\mathbf{9}$. An oven-dried, two-neck reaction flask was charged with 1,1'-di-tert-butylferrocene ${ }^{[12]}(7 ; 1.0 \mathrm{~g}, 3.35 \mathrm{mmol})$ and anhydrous pentane (5 mL). Distilled $N, N, N^{\prime}, N^{\prime}$-tetramethyl-1,2- 
Lorsqu'un écrit scientifique issu d'une activité de recherche financée au moins pour moitié par des dotations de l'État, des collectivités territoriales ou des établissements publics, par des subventions d'agences de financement nationales ou par des fonds de l'Union européenne est publié dans un périodique paraissant au moins une fois par an, son auteur dispose, même après avoir accordé des droits exclusifs à un éditeur, du droit de mettre à disposition gratuitement dans un format ouvert, par voie numérique, sous réserve de l'accord des éventuels coauteurs, la version finale de son manuscrit acceptée pour publication, dès lors que l'éditeur met lui-même celle-ci gratuitement à disposition par voie numérique ou, à défaut, à l'expiration d'un délai courant à compter de la date de la première publication. Ce délai est au maximum de six mois pour une publication dans le domaine des sciences, de la technique et de la médecine et de douze mois dans celui des sciences humaines et sociales.

diaminoethane $(1 \mathrm{~mL}, 6.7 \mathrm{mmol})$ was added and the resulting solution was cooled at $-80^{\circ} \mathrm{C}$. tert-Butyllithium $(4.0 \mathrm{~mL}$ of $1.7 \mathrm{M}$ solution in pentane, 6.8 $\mathrm{mmol}$ ) was introduced and the reaction mixture was warmed up to room temperature while stirring overnight. Then, the mixture was filtered and the solid was washed twice with pentane to obtain adduct 1,1'-dilithio-3,3'-ditert-butylferrocene- $N, N, N^{\prime}, N^{\prime}$ 'tetramethyl-1,2-diaminoethane (8) as an orange solid $(57 \%$ yield, $813 \mathrm{mg})$. Next, adduct $8(812.6 \mathrm{mg}, 1.91 \mathrm{mmol})$ and anhydrous pentane $(14.5 \mathrm{ml})$ were cooled at $-80^{\circ} \mathrm{C}$. Dichlorophenylphosphane $(0.27 \mathrm{~mL}, 2.0 \mathrm{mmol})$ in pentane $(18.3 \mathrm{~mL})$ was introduced drop by drop and the reaction mixture was warmed up to room temperature overnight. Following quenching with distilled water $(15 \mathrm{~mL})$, the organic layer was separated, washed with distilled water, dried over anhydrous magnesium sulfate and, finally, evaporated. Crude product was obtained as a mixture of $89 \%$ of 3,3'-di-tert-butyl,1-phenyl-1'phosphaferrocenophane (9), 7\% of 1,1'-di-tert-butylferrocene (7) and $4 \%$ of $N, N, N^{\prime}, N^{\prime}$-tetramethyl-1,2-diaminoethane (NMR yields) and was not further purified.

For 9: ${ }^{1} \mathrm{H}$ NMR $\left(500 \mathrm{MHz}, \mathrm{CDCl}_{3}\right): \delta 7.64(2 \mathrm{H}$, ddd, $J=8.0,6.5,1.3 \mathrm{~Hz})$, $7.42(2 \mathrm{H}, \mathrm{td}, J=7.7,2.0 \mathrm{~Hz}), 7.35-7.27(1 \mathrm{H}, \mathrm{m}), 4.50(2 \mathrm{H}, \mathrm{q}, J=1.4$ $\mathrm{Hz}), 4.32(1 \mathrm{H}, \mathrm{dd}, J=2.4,1.3 \mathrm{~Hz}), 4.30(1 \mathrm{H}, \mathrm{dt}, J=2.3,1.0 \mathrm{~Hz}), 4.28(1$ $\mathrm{H}, \mathrm{dd}, J=2.4,1.4 \mathrm{~Hz}), 4.25(1 \mathrm{H}, \mathrm{t}, J=1.4 \mathrm{~Hz}), 1.19(9 \mathrm{H}, \mathrm{s}), 1.13(9 \mathrm{H}$, s). ${ }^{31} \mathrm{P}\left\{{ }^{1} \mathrm{H}\right\} \mathrm{NMR}\left(202 \mathrm{MHz}, \mathrm{CDCl}_{3}\right): \delta-15.14(\mathrm{~s})$. Impurities did not allow unambiguous assignment of the ${ }^{13} \mathrm{C}$ NMR signals.

Compound 9 ( $683.3 \mathrm{mg}, 1.69 \mathrm{mmol}, \approx 90 \%$ pure) and diethyl ether $(10 \mathrm{~mL})$ was cooled at $-80^{\circ} \mathrm{C}$. Phenyllithium $(7.5 \mathrm{~mL}$ of $0.39 \mathrm{M}$ solution in diethyl ether, $2.93 \mathrm{mmol}$ ) was introduced dropwise and the mixture was stirred for 30 min with continuous cooling and then $40 \mathrm{~min}$ at room temperature. After carbon dioxide gas was bubbled through the solution of the lithiated intermediate with stirring at $0{ }^{\circ} \mathrm{C}$ for $6 \mathrm{~h}$, the mixture was quenched with saturated $5 \%$ aqueous $\mathrm{NaOH}$. The crude product obtained after acidification was found difficult to purify. Hence, pure $\mathbf{1 0}$ was obtained by using a procedure similar to the synthesis of $\mathbf{1 9}$ described below. Yield: $44.0 \mathrm{mg}(5 \%)$; for analytical data see above.

Synthesis of 10 by deborylation of adduct 18 . Under argon, compound $18(0.15 \mathrm{~g}, 0.27 \mathrm{mmol})$ was dissolved in degassed absolute ethanol (5.5 $\mathrm{mL}$ ) and the solution was heated at reflux for $10 \mathrm{~h}$ under argon. The reaction mixture was cooled to room temperature and concentrated under vacuum. The crude product was purified by column chromatography (silica gel, hexane-ethyl acetate 5:1). First minor band was discarded and the following orange band was collected and evaporated to afford deprotected acid 10 as an orange solid. Yield: $128 \mathrm{mg}(90 \%)$. The analytical data were identical to those given above.

Synthesis of 10Se from 10. An oven-dried, two-neck reaction flask was charged with 1'-(diphenylphosphanyl)-3,3'-di-tert-butylferrocene-1carboxylic acid (10; $26.3 \mathrm{mg}, 0.05 \mathrm{mmol})$ and an excess of selenium black $(60 \mathrm{mg}, 0.76 \mathrm{mmol})$ in anhydrous dichloromethane $(0.5 \mathrm{~mL})$. The mixture was stirring for $24 \mathrm{~h}$ at room temperature then the solution was filtered to obtain 10Se in quantitative yield as testified by multinuclear NMR analysis. Crystal used for structure determination was obtained from $\mathrm{CH}_{2} \mathrm{Cl}_{2}-$ heptane.

${ }^{1} \mathrm{H} \mathrm{NMR}\left(500 \mathrm{MHz}, \mathrm{CDCl}_{3}\right): \delta 7.84-7.76(2 \mathrm{H}, \mathrm{m}, \mathrm{Ph}), 7.61-7.51(2 \mathrm{H}$ $\mathrm{m}, \mathrm{Ph}), 7.51-7.42(3 \mathrm{H}, \mathrm{m}, \mathrm{Ph}), 7.42-7.32(3 \mathrm{H}, \mathrm{m}, \mathrm{Ph}), 4.76-4.71$ (1 $\mathrm{H}, \mathrm{m}, \mathrm{Cp}), 4.64(1 \mathrm{H}, \mathrm{t}, J=1.5 \mathrm{~Hz}, \mathrm{Cp}), 4.51(1 \mathrm{H}, \mathrm{dt}, J=3.0,1.5 \mathrm{~Hz}, \mathrm{Cp})$, $4.42(1 \mathrm{H}, \mathrm{q}, J=2.1 \mathrm{~Hz}, \mathrm{Cp}), 4.33(1 \mathrm{H}, \mathrm{dd}, J=2.7,1.6 \mathrm{~Hz}, \mathrm{Cp}), 4.31(1 \mathrm{H}$, $\mathrm{dt}, J=2.8,1.6 \mathrm{~Hz}, \mathrm{Cp}), 1.33(9 \mathrm{H}, \mathrm{s}, t-\mathrm{Bu}), 0.99(9 \mathrm{H}, \mathrm{s}, t-\mathrm{Bu}) \cdot{ }^{13} \mathrm{C}\left\{{ }^{1} \mathrm{H}\right\} \mathrm{NMR}$ (126 MHz, $\mathrm{CDCl}_{3}$ ): $\delta$ 177.22, 134.49 (d, $J=78.5 \mathrm{~Hz}$ ), 133.47 (d, $J=77.7$ Hz), 132.58 (d, $J=10.9 \mathrm{~Hz}), 132.04(\mathrm{~d}, J=10.9 \mathrm{~Hz}), 131.48(\mathrm{~d}, J=2.9$ $\mathrm{Hz}), 131.20(\mathrm{~d}, J=2.9 \mathrm{~Hz}), 128.43(\mathrm{~d}, J=3.0 \mathrm{~Hz}), 128.33(\mathrm{~d}, J=3.0 \mathrm{~Hz})$, $108.05(\mathrm{~d}, J=7.9 \mathrm{~Hz}), 74.90,74.21,73.98(\mathrm{~d}, J=26.9 \mathrm{~Hz}), 72.60(\mathrm{~d}, J=$
$10.9 \mathrm{~Hz}), 72.04,71.27,70.70,70.43,69.28,31.79,31.73,31.13,30.49$. ${ }^{31} \mathrm{P}\left\{{ }^{1} \mathrm{H}\right\}$ NMR $\left(202 \mathrm{MHz}, \mathrm{CDCl}_{3}\right): \delta 31.35\left({ }^{1} \mathrm{JPSe}=736 \mathrm{~Hz}\right)$. Anal. Calc. for $\mathrm{C}_{31} \mathrm{H}_{35} \mathrm{FeO}_{2} \mathrm{PSe} \cdot 1.7$ dichloromethane $\cdot 0.8$ heptane (844.45): C $55.43 \%$, $\mathrm{H} 6.22 \%$. Found C $55.43 \%, \mathrm{H} 6.22 \%$. ESI+ MS calc. for $\mathrm{C}_{31} \mathrm{H}_{35} \mathrm{FeO}_{2} \mathrm{PSe}$ $\left([\mathrm{M}+\mathrm{H}]^{+}\right): 629.0787$, found: 629.0762 .

Synthesis of 15. An oven-dried, two-neck reaction flask was charged with 1,1'-dibromo-3,3'-di-tert-butylferrocene (11; $0.46 \mathrm{~g}, 1.0 \mathrm{mmol})$ and anhydrous THF $(8 \mathrm{~mL})$ and the resulting solution was cooled with a dry ice/ethanol bath to ca. $-75^{\circ} \mathrm{C}$. $n$-Butyllithium $(0.63 \mathrm{~mL}$ of $1.6 \mathrm{M}$ solution in hexane, $1.0 \mathrm{mmol}$ ) was introduced and the mixture was stirred for $30 \mathrm{~min}$ with continuous cooling. Chlorodiphenylphosphane $(0.20 \mathrm{~mL}, 1.1 \mathrm{mmol})$ to the lithiated intermediate and the stirring was continued at $-75^{\circ} \mathrm{C}$ for 30 min and at room temperature for additional 90 min. Next, neat $\mathrm{BH}_{3} \cdot \mathrm{SMe}_{2}$ $(0.11 \mathrm{~mL}, 1.2 \mathrm{mmol})$ was added and the mixture was stirred for $30 \mathrm{~min}$ before quenching with saturated aqueous $\mathrm{NaHCO}_{3}$ (Caution! Gas evolution.). The mixture was partitioned between diethyl ether $(20 \mathrm{~mL})$ and brine $(10 \mathrm{~mL})$. The organic layer was separated, washed with brine, dried over anhydrous magnesium sulfate and evaporated with chromatographic silica gel to afford crude product in pre-adsorbed form. The crude product was transferred to the top of a silica gel column. Elution with hexanediethyl ether $(5: 1)$ removed a minor yellow band containing the starting dibromide and non-polar by-products. Changing the eluent to a hexanediethyl ether 1:1 mixture led to the development of a major band due to the product, which was collected and evaporated to afford analytically pure adduct 15 as an orange yellow solid $(0.45 \mathrm{~g}, 78 \%)$.

${ }^{1} \mathrm{H} \mathrm{NMR}\left(400 \mathrm{MHz}, \mathrm{CDCl}_{3}\right): \delta 7.65-7.57(2 \mathrm{H}, \mathrm{m}, \mathrm{Ph}), 7.50-7.33(8 \mathrm{H}, \mathrm{m}$, $\mathrm{Ph}), 4.53(1 \mathrm{H}, \mathrm{td}, J=2.5,1.4 \mathrm{~Hz}, \mathrm{Cp}), 4.36(1 \mathrm{H}, \mathrm{t}, J=1.5 \mathrm{~Hz}, 1 \mathrm{H}, \mathrm{Cp})$, 4.28-4.26 (2 H, m, Cp), $4.04(1 \mathrm{H}, \mathrm{dt}, J=2.3,1.4 \mathrm{~Hz}, \mathrm{Cp}), 3.97(1 \mathrm{H}, \mathrm{dd}$, $J 2.5,1.6, \mathrm{Cp}), 1.32(9 \mathrm{H}, \mathrm{s}, t-\mathrm{Bu}), 1.02(9 \mathrm{H}, \mathrm{s}, t-\mathrm{Bu}), 1.70-0.80(3 \mathrm{H}, \mathrm{br}$ $\left.\mathrm{m}, \mathrm{BH}_{3}\right) .{ }^{13} \mathrm{C}\left\{{ }^{1} \mathrm{H}\right\} \mathrm{NMR}\left(101 \mathrm{MHz}, \mathrm{CDCl}_{3}\right): \delta 133.35(\mathrm{~d}, J=9.8 \mathrm{~Hz}), 132.59$ $(\mathrm{d}, J=9.5 \mathrm{~Hz}), 132.36(\mathrm{~d}, J=59.2 \mathrm{~Hz}), 131.03(\mathrm{~d}, J=59.2 \mathrm{~Hz}), 130.92(\mathrm{~d}$, $J=2.5 \mathrm{~Hz}), 130.64(\mathrm{~d}, J=2.4 \mathrm{~Hz}), 128.53(\mathrm{~d}, J=10.1 \mathrm{~Hz}), 128.38(\mathrm{~d}, J=$ $10.1 \mathrm{~Hz}), 107.57(\mathrm{~d}, J=6.4 \mathrm{~Hz}), 102.61,77.42,73.79(\mathrm{~d}, J=7.3 \mathrm{~Hz}), 73.35$ (d, $J=10.7 \mathrm{~Hz}), 71.21,70.14(\mathrm{~d}, J=66.5 \mathrm{~Hz}), 68.93,65.03,30.60,31.50$, 30.99, 30.17. ${ }^{31} \mathrm{P}\left\{{ }^{1} \mathrm{H}\right\}$ NMR (162 MHz, $\mathrm{CDCl}_{3}$ ): $\delta 17.08$ (br d). IR (Nujol): $v_{\max } 2409 \mathrm{~s}, 2392 \mathrm{~s}, 2358 \mathrm{~m}, 2256 \mathrm{w}, 1484 \mathrm{~m}, 1435 \mathrm{~s}, 1316 \mathrm{w}, 1283 \mathrm{~m}$, 1275 m, 1214 w, 1199 w, 1182 m, 1134 m, 1109 m, 1184 w, 1064 s, 1047 w, 1039 w, 1027 w, 999 w, 927 m, 918 w, 881 m, 846 m, 822 w, 810 m, $766 \mathrm{w}, 739$ s, $704 \mathrm{~m}, 696 \mathrm{~m}, 689 \mathrm{w}, 675 \mathrm{w}, 643 \mathrm{~m}, 595 \mathrm{~m}, 531 \mathrm{w}, 511 \mathrm{~m}$, 504 w, 490 m, 476 m, 445 w, $430 \mathrm{~m} \mathrm{~cm}^{-1}$. Anal. Calc. for $\mathrm{C}_{30} \mathrm{H}_{37} \mathrm{FeBBrP}$ (575.15): C 62.65, H 6.48\%. Found: C 62.63, H 6.40\%. ESI+ MS calc. for $\mathrm{C}_{30} \mathrm{H}_{37} \mathrm{FeBBrNaP}\left([\mathrm{M}+\mathrm{Na}]^{+}\right): 597.1151$, found: 597.1154 .

Synthesis of 16. An oven-dried, two-neck reaction flask was charged with 1,1'-dibromo-3,3'-di-tert-butylferrocene $(\mathbf{1 1} ; 0.5 \mathrm{~g}, 1.1 \mathrm{mmol})$ and anhydrous THF $(3.2 \mathrm{~mL})$ and the resulting solution was cooled at $-80^{\circ} \mathrm{C}$. $n$-Butyllithium $(0.68 \mathrm{~mL}$ of $1.6 \mathrm{M}$ solution in hexane, $1.08 \mathrm{mmol})$ was introduced and the mixture was stirred for $1 \mathrm{~h}$ with continuous cooling. Chlorodicyclohexylphosphane $(0.24 \mathrm{~mL}, 1.08 \mathrm{mmol})$ in anhydrous THF $(1.3 \mathrm{~mL})$ was added to the lithiated intermediate and the stirring was continued at room temperature overnight. Then, $\mathrm{BH}_{3} \cdot \mathrm{SMe}_{2}(0.7 \mathrm{~mL}$ of 2.0 $\mathrm{M}$ solution in THF, $1.4 \mathrm{mmol}$ ) was added and the mixture was stirred for additional $1 \mathrm{~h}$ before quenching with saturated aqueous $\mathrm{NaHCO}_{3}$. The reaction mixture was partitioned between diethyl ether $(20 \mathrm{~mL})$ and brine $(10 \mathrm{~mL})$. The organic layer was separated, washed with brine, dried over anhydrous magnesium sulfate and evaporated. The crude product was purified by chromatography over a silica gel column using hexanedichloromethane (4:1) as the eluent. The first minor band was discarded containing the starting 1,1'-di-tert-butylferrocene and the following major 
Lorsqu'un écrit scientifique issu d'une activité de recherche financée au moins pour moitié par des dotations de l'État, des collectivités territoriales ou des établissements publics, par des subventions d'agences de financement nationales ou par des fonds de l'Union européenne est publié dans un périodique paraissant au moins une fois par an, son auteur dispose, même après avoir accordé des droits exclusifs à un éditeur, du droit de mettre à disposition gratuitement dans un format ouvert, par voie numérique, sous réserve de l'accord des éventuels coauteurs, la version finale de son manuscrit acceptée pour publication, dès lors que l'éditeur met lui-même celle-ci gratuitement à disposition par voie numérique ou, à défaut, à l'expiration d'un délai courant à compter de la date de la première publication. Ce délai est au maximum de six mois pour une publication dans le domaine des sciences, de la technique et de la médecine et de douze mois dans celui des sciences humaines et sociales.

one collected and evaporated to give compound 16 as an orange solid. Yield: $0.34 \mathrm{~g}(54 \%)$.

${ }^{1} \mathrm{H}$ NMR $\left(600 \mathrm{MHz}, \mathrm{CDCl}_{3}\right): \delta 4.47(2 \mathrm{H}, \mathrm{s}, \mathrm{Cp}), 4.38(1 \mathrm{H}, \mathrm{s}, \mathrm{Cp}), 4.19(1$ $\mathrm{H}, \mathrm{s}, \mathrm{Cp}), 4.05(1 \mathrm{H}, \mathrm{s}, \mathrm{Cp}), 3.87(1 \mathrm{H}, \mathrm{s}, \mathrm{Cp}), 2.28-2.12(2 \mathrm{H}, \mathrm{m}, \mathrm{Cy})$, $1.99(1 \mathrm{H}, \mathrm{d}, J=13 \mathrm{~Hz}, \mathrm{Cy}), 1.93-1.81(3 \mathrm{H}, \mathrm{m}, \mathrm{Cy}), 1.80-1.66(4 \mathrm{H}, \mathrm{m}$ Cy), $1.64(1 \mathrm{H}, \mathrm{d}, J=12.5 \mathrm{~Hz}, \mathrm{Cy}), 1.50-1.31(4 \mathrm{H}, \mathrm{m}, \mathrm{Cy}), 1.29(9 \mathrm{H}, \mathrm{s}$, $t$-Bu), $1.27-1.23(3 \mathrm{H}, \mathrm{m}, \mathrm{Cy}), 1.22(9 \mathrm{H}, \mathrm{s}, t-\mathrm{Bu}), 1.20-0.99(4 \mathrm{H}, \mathrm{m}$, Cy), $0.78-0.28\left(3 \mathrm{H}, \mathrm{br} m, \mathrm{BH}_{3}\right) .{ }^{13} \mathrm{C}\left\{{ }^{1} \mathrm{H}\right\} \mathrm{NMR}\left(151 \mathrm{MHz}, \mathrm{CDCl}_{3}\right): \delta 106.48$ $(\mathrm{d}, J=5.0 \mathrm{~Hz}), 102.50,78.44,74.38(\mathrm{~d}, J=10.7 \mathrm{~Hz}), 72.44(\mathrm{~d}, J=3.6 \mathrm{~Hz})$, $71.22(\mathrm{~d}, J=55.0 \mathrm{~Hz}), 71.18,69.15,68.98(\mathrm{br} \mathrm{m}), 64.81,33.83(\mathrm{~d}, J=33.7$ $\mathrm{Hz}), 32.76(\mathrm{~d}, J=34.6 \mathrm{~Hz}), 32.03,31.96,31.64,31.06,30.67,28.20,27.72$ (d, $J=2.5 \mathrm{~Hz}$ ), 27.57, 27.34, 27.33, 27.31, 27.26, 27.07, 27.03, 27.00, 27.00, 26.95, 26.23, 26.18, 22.84. ${ }^{31} \mathrm{P}\left\{{ }^{1} \mathrm{H}\right\} \mathrm{NMR}\left(243 \mathrm{MHz}, \mathrm{CDCl}_{3}\right): \delta 24.70$ (d, $\left.{ }^{1} \mathrm{~J}_{\mathrm{BP}}=71.8 \mathrm{~Hz}\right) \cdot{ }^{11} \mathrm{~B}\left\{{ }^{1} \mathrm{H}\right\} \mathrm{NMR}\left(193 \mathrm{MHz}, \mathrm{CDCl}_{3}\right): \delta-42.26\left(\mathrm{t},{ }^{1} \mathrm{JPB}=\right.$ $71.25 \mathrm{~Hz}$ ). Anal. Calc. for $\mathrm{C}_{30} \mathrm{H}_{49} \mathrm{BBrFeP} \cdot 0.5 \mathrm{H}_{2} \mathrm{O} \cdot 0.3$ hexane (634.68): C $60.18 \%, \mathrm{H} 8.83 \%$. Found C $59.94 \%, \mathrm{H} 9.00 \%$. ESI+ MS calc. for $\mathrm{C}_{30} \mathrm{H}_{49} \mathrm{BBrFeNaP}\left([\mathrm{M}+\mathrm{Na}]^{+}\right): 609.2095$, found: 609.2106

Synthesis of 17. An oven-dried, two-neck reaction flask was charged with 1,1'-dibromo-3,3'-di-tert-butylferrocene (11; $3.23 \mathrm{~g}, 7.09 \mathrm{mmol})$ and anhydrous THF $(20.8 \mathrm{~mL})$ and the resulting solution was cooled at $-80^{\circ} \mathrm{C}$. $n$-Butyllithium (4.34 mL of $1.6 \mathrm{M}$ solution in hexane, $6.95 \mathrm{mmol}$ ) was introduced and the mixture was stirred for $1 \mathrm{~h}$ with continuous cooling. Chloro-diisopropylphosphane $(1.1 \mathrm{~mL}, 6.95 \mathrm{mmol})$ in anhydrous THF $(7.0$ $\mathrm{mL}$ ) was added to the lithiated intermediate and the stirring was continued at room temperature overnight. The mixture was partitioned between dichloromethane and brine. The organic layer was separated washed with brine, dried over anhydrous magnesium sulfate and finally evaporated. The crude product was purified by chromatography over silica gel column using hexane-dichloromethane (11:9) as the eluent. First minor band was discarded and the following orange band was collected and evaporated to give compound 14 as an orange solid. Yield: $2.402 \mathrm{~g}(71 \%)$.

In the following step, an oven-dried, two-neck reaction flask was charged with 1-(diisopropylphosphanyl)-1'-bromo-3,3'-di-tert-butylferrocene (14; $1.09 \mathrm{~g}, 2.21 \mathrm{mmol}$ ) and anhydrous dichloromethane $(20 \mathrm{~mL})$. $\mathrm{BH}_{3} \cdot \mathrm{SMe}_{2}$ $(1.66 \mathrm{~mL}$ of $2.0 \mathrm{M}$ solution in THF, $3.32 \mathrm{mmol}$ ) was added and the mixture was stirred for $1.5 \mathrm{~h}$ before quenching with distilled water. The mixture was partitioned between dichloromethane and brine. The organic layer was separated, washed with brine, dried over anhydrous magnesium sulfate and, finally, evaporated. The obtained crude product was purified by recrystallization from hot heptane to give compound $\mathbf{1 7}$ as orange crystals. Yield: $0.67 \mathrm{~g}(60 \%)$.

${ }^{1} \mathrm{H}$ NMR $\left(600 \mathrm{MHz}, \mathrm{CDCl}_{3}\right): \delta 4.52(1 \mathrm{H}, \mathrm{s}, \mathrm{Cp}), 4.47(1 \mathrm{H}, \mathrm{s}, \mathrm{Cp}), 4.39$ (1 $\mathrm{H}, \mathrm{s}, \mathrm{Cp}), 4.19(1 \mathrm{H}, \mathrm{s}, \mathrm{Cp}), 4.06(1 \mathrm{H}, \mathrm{s}, \mathrm{Cp}), 3.4(1 \mathrm{H}, \mathrm{s}, \mathrm{Cp}), 2.51-2.42$ $(1 \mathrm{H}, \mathrm{d}$ of sept, $J=7.0,11.4 \mathrm{~Hz}, i-\mathrm{Pr}), 2.11-2.03(1 \mathrm{H}, \mathrm{d}$ of sept, $J=6.7$, $10.7 \mathrm{~Hz}, i-\mathrm{Pr}), 1.35-1.27(6 \mathrm{H}, \mathrm{m}, i-\mathrm{Pr}), 1.29(9 \mathrm{H}, \mathrm{s}, t-\mathrm{Bu}), 1.22(9, \mathrm{H}, \mathrm{s}, t-$ $\mathrm{Bu}), 1.15-1.11(3 \mathrm{H}, \mathrm{dd}, J=7.0,14.9 \mathrm{~Hz}, i-\operatorname{Pr}), 0.95-0.92(3 \mathrm{H}, \mathrm{dd}, J=$ 7.0, $14.0 \mathrm{~Hz}, i-\mathrm{Pr}), 0.84-0.29\left(3 \mathrm{H}, \mathrm{br} \mathrm{m}, \mathrm{BH}_{3}\right) .{ }^{13} \mathrm{C}\left\{{ }^{1} \mathrm{H}\right\} \mathrm{NMR}(151 \mathrm{MHz}$, $\left.\mathrm{CDCl}_{3}\right): \delta 106.71(\mathrm{~d}, J=4.4 \mathrm{~Hz}), 102.6,78.3,74.28(\mathrm{~d}, J=11.3 \mathrm{~Hz}), 72.23$ $(\mathrm{d}, J \mathrm{PC}=3.9 \mathrm{~Hz}), 71.12(\mathrm{~d}, J=55.1 \mathrm{~Hz}), 71.11,69.48(\mathrm{br} \mathrm{s}), 69.04,64.99$, $31.95,31.65,31.03,30.66,23.97(\mathrm{~d}, J=33.7 \mathrm{~Hz}), 23.75(\mathrm{~d}, J=35.0 \mathrm{~Hz})$, 18.95, $18.16(\mathrm{~d}, J=2.2 \mathrm{~Hz}), 17.77 .{ }^{31} \mathrm{P}\left\{{ }^{1} \mathrm{H}\right\} \operatorname{NMR}\left(243 \mathrm{MHz}, \mathrm{CDCl}_{3}\right): \delta$ $31.41\left(\mathrm{~d},{ }^{1} \mathrm{~J}_{\mathrm{BP}}=73.4 \mathrm{~Hz}\right) .{ }^{11} \mathrm{~B} \mathrm{NMR}\left(193 \mathrm{MHz}, \mathrm{CDCl}_{3}\right): \delta-42.83\left(\mathrm{~m},{ }^{1} \mathrm{JPB}_{\mathrm{PB}}=\right.$ $76.6 \mathrm{~Hz}$ ). Anal. Calc. for $\mathrm{C}_{24} \mathrm{H}_{41} \mathrm{BBrFeP}$ (507.12): C $56.84 \%$, H 8.15\%. Found C $56.92 \%, \mathrm{H} 8.47 \%$. ESI+ MS calc. for $\mathrm{C}_{24} \mathrm{H}_{41} \mathrm{BBrFeNaP}([\mathrm{M}+$ $\left.\mathrm{Na}^{+}\right)$: 529.1469 , found: 529.1473 .

Synthesis of 18 . Adduct $15(0.58 \mathrm{~g}, 1.0 \mathrm{mmol})$ was dissolved in anhydrous THF (15 mL) in an oven-dried reaction flask. The stirring solution was cooled to approximately $-75^{\circ} \mathrm{C}$ with a dry ice/ethanol bath and treated with $n$-butyllithium ( $0.69 \mathrm{~mL}$ of $1.6 \mathrm{M}$ in hexane, $1.1 \mathrm{mmol}$ ). After stirring for 30 min, crushed dry ice (ca. $40 \mathrm{~g}$ ) was added and the mixture was allowed to warm to room temperature during $90 \mathrm{~min}$. Next, it was acidified with methanolic $\mathrm{HCl}$ (1.76 mL of $1.25 \mathrm{M}$ solution) and concentrated under reduced pressure. The oily residue was taken up with dichloromethane (20 $\mathrm{mL})$ and the solution washed with brine $(2 \times 15 \mathrm{~mL})$, dried over $\mathrm{MgSO}_{4}$ and evaporated. The crude product was purified by chromatography over a silica gel column using dichloromethane-methanol $(50: 1)$ as the eluent. The first minor band was discarded and the following major one collected and evaporated to give compound 18 as an orange powder. Yield: $0.42 \mathrm{~g}$ (78\%). Crystals used for structure determination were obtained from chloroform-hexane.

${ }^{1} \mathrm{H}$ NMR $\left(400 \mathrm{MHz}, \mathrm{CDCl}_{3}\right): \delta 7.60-7.54(2 \mathrm{H}, \mathrm{m}, \mathrm{Ph}), 7.52-7.32(8 \mathrm{H}$, $\mathrm{m}, \mathrm{Ph}), 4.74(1 \mathrm{H}, \mathrm{dd}, J=2.6,1.4 \mathrm{~Hz}, \mathrm{Cp}), 4.62(1 \mathrm{H}, \mathrm{t}, J=1.5 \mathrm{~Hz}, \mathrm{Cp})$, $4.47(1 \mathrm{H}, \mathrm{td}, J=2.4,1.4 \mathrm{~Hz}, \mathrm{Cp}), 4.34(1 \mathrm{H}, \mathrm{dd}, J=2.7,1.6 \mathrm{~Hz}, \mathrm{Cp}), 4.32$ - $4.28(2 \mathrm{H}, \mathrm{m}, \mathrm{Cp}), 1.34(9 \mathrm{H}, \mathrm{s}, t-\mathrm{Bu}), 1.02(9 \mathrm{H}, \mathrm{s}, t-\mathrm{Bu}), 1.60-0.80$ (3 $\left.\mathrm{H}, \mathrm{br} \mathrm{m}, \mathrm{BH}_{3}\right) .{ }^{13} \mathrm{C}\left\{{ }^{1} \mathrm{H}\right\} \mathrm{NMR}\left(101 \mathrm{MHz}, \mathrm{CDCl}_{3}\right): \delta 176.94,132.84(\mathrm{~d}, J=$ $9.5 \mathrm{~Hz}$ ), $132.77(\mathrm{~d}, J=9.8 \mathrm{~Hz}), 131.67(\mathrm{~d}, J=58.6 \mathrm{~Hz}), 131.14(\mathrm{~d}, J=59.2$ $\mathrm{Hz}), 130.93(\mathrm{~d}, J=2.5 \mathrm{~Hz}), 130.80(\mathrm{~d}, J=2.4 \mathrm{~Hz}), 128.51(\mathrm{~d}, J=4.9 \mathrm{~Hz})$, $128.41(\mathrm{~d}, J=4.9 \mathrm{~Hz}), 107.98(\mathrm{~d}, J=6.4 \mathrm{~Hz}), 107.87,72.75(\mathrm{~d}, J=8.2$ $\mathrm{Hz}$ ), 71.82, 71.77 (d, $J=9.2 \mathrm{~Hz}$ ), 70.94 (br s), 70.20, 70.08 (d, $J=65.3$ $\mathrm{Hz}), 69.86,68.85,31.66,31.56,30.97,30.35 .{ }^{31} \mathrm{P}\left\{{ }^{1} \mathrm{H}\right\} \mathrm{NMR}(162 \mathrm{MHz}$, $\mathrm{CDCl}_{3}$ ): $\delta 17.21$ (br s). IR (Nujol): $v_{\max } 2637 \mathrm{~m}, 2601 \mathrm{~m}, 2554 \mathrm{~m}, 2397 \mathrm{~m}$ $2369 \mathrm{~m}, 2341 \mathrm{~m}, 1675$ vs $(\mathrm{COOH}), 1490 \mathrm{~m}, 1479 \mathrm{~s}, 1435 \mathrm{~m}, 1396 \mathrm{w}, 1342$ w, $1311 \mathrm{~m}, 1261 \mathrm{~s}, 1218 \mathrm{w}, 1199 \mathrm{w}, 1180 \mathrm{~m}, 1132 \mathrm{w}, 1109 \mathrm{~m}, 1077 \mathrm{~m}$ 1058 m, 1047 w, 1036 w, 1028 w, 999 w, 967 m, 940 w, 922 m, 903 w, $884 \mathrm{w}, 862 \mathrm{~m}, 853 \mathrm{~m}, 837 \mathrm{w}, 830 \mathrm{w}, 816 \mathrm{w}, 787 \mathrm{w}, 752 \mathrm{~m}, 744 \mathrm{~m}, 736 \mathrm{~m}$ $701 \mathrm{~m}, 694 \mathrm{~m}, 673 \mathrm{w}, 639 \mathrm{~m}, 622 \mathrm{w}, 613 \mathrm{w}, 593 \mathrm{w}, 564 \mathrm{~m}, 530 \mathrm{w}, 511 \mathrm{~m}$, $492 \mathrm{~m}, 472 \mathrm{~m}, 444 \mathrm{w}, 432 \mathrm{w} \mathrm{cm}^{-1}$. Anal. Calc. for $\mathrm{C}_{31} \mathrm{H}_{38} \mathrm{FeBO} \mathrm{Fe}_{2} \mathrm{P}(540.26)$ : C $68.92, \mathrm{H} 7.09 \%$. Found: C $68.79, \mathrm{H} 7.23 \%$. ESI+ MS calc. for $\mathrm{C}_{31} \mathrm{H}_{38} \mathrm{FeBO}{ }_{2} \mathrm{NaP}\left([\mathrm{M}+\mathrm{Na}]^{+}\right): 563.1944$, found: 563.1947 .

Synthesis of 19. An oven-dried, two-neck reaction flask was charged with 1-(dicyclohexylphosphanyl)-1'-bromo-3,3'-di-tert-butylferrocene-borane (16; $51.9 \mathrm{mg}, 0.09 \mathrm{mmol})$ in anhydrous diethyl ether $(1.5 \mathrm{~mL})$ and the resulting solution was cooled at $-80^{\circ} \mathrm{C}$. $n$-Butyllithium $(0.09 \mathrm{~mL}$ of $1.6 \mathrm{M}$ solution in hexane, $0.14 \mathrm{mmol}$ ) was introduced and the mixture was stirred for $1 \mathrm{~h}$ with continuous cooling. Next, the carbon dioxide gas was passed through the reaction mixture while stirring and cooling to $0{ }^{\circ} \mathrm{C}$ for $6 \mathrm{~h}$, before quenching with $5 \%$ aqueous $\mathrm{NaOH}$. The mixture was partitioned between diethyl ether and distilled water. The organic layer was separated, washed with brine, dried over anhydrous magnesium sulfate and, finally, evaporated. The aqueous layer was collected and few drops of $6 \mathrm{M} \mathrm{HCl}$ were added to obtain a red precipitate. The red precipitate was dissolved in dichloromethane, and the solution was dried over $\mathrm{MgSO}_{4}$ and evaporated. The residue was purified by chromatography over silica gel column using dichloromethane-methanol (99:1) as the eluent. First minor band was the 1-(dicyclohexylphosphanyl)-3,3'-di-tert-butylferrocene and the following band was collected and evaporated to give compound 19 as an orange solid. Yield: $19 \mathrm{mg}(38 \%)$.

${ }^{1} \mathrm{H}$ NMR $\left(500 \mathrm{MHz}, \mathrm{CDCl}_{3}\right): \delta 4.88(1 \mathrm{H}, \mathrm{dd}, J=2.6,1.3 \mathrm{~Hz}, \mathrm{Cp}), 4.78(1$ $\mathrm{H}, \mathrm{t}, J=1.5 \mathrm{~Hz}, \mathrm{Cp}), 4.44(1 \mathrm{H}, \mathrm{d}, J=2.6,1.6 \mathrm{~Hz}, \mathrm{Cp}), 4.40(1 \mathrm{H}, \mathrm{dt}, J=$ 2.9, $1.5 \mathrm{~Hz}, \mathrm{Cp}), 4.23(1 \mathrm{H}, \mathrm{q}, J=1.5 \mathrm{~Hz}, \mathrm{Cp}), 4.17(1 \mathrm{H}, \mathrm{dt}, J=2.5,1.3$ $\mathrm{Hz}, \mathrm{Cp}$ ), $2.03-1.89(4 \mathrm{H}, \mathrm{m}, \mathrm{Cy}), 1.86-1.73(4 \mathrm{H}, \mathrm{m}, \mathrm{Cy}), 1.72-1.61$ (3 $\mathrm{H}, \mathrm{m}, \mathrm{Cy}), 1.44-1.34(3 \mathrm{H}, \mathrm{m}, \mathrm{Cy}), 1.30(9 \mathrm{H}, \mathrm{s}, t-\mathrm{Bu}), 1.28(9 \mathrm{H}, \mathrm{s}, t-\mathrm{Bu})$, $1.25-1.07(6 \mathrm{H}, \mathrm{m}, \mathrm{Cy}), 0.79-0.2\left(3 \mathrm{H}, \mathrm{br} \mathrm{m}, \mathrm{BH}_{3}\right) .{ }^{13} \mathrm{C}\left\{{ }^{1} \mathrm{H}\right\} \mathrm{NMR}(126$ $\left.\mathrm{MHz}, \mathrm{CDCl}_{3}\right): \delta 177.52(\mathrm{~s}, \mathrm{COOH}), 107.65,106.67(\mathrm{~d}, J=5.6 \mathrm{~Hz}), 73.36$ (br m), 72.16, 71.24 (d, $J=53.6 \mathrm{~Hz}), 70.93(\mathrm{~d}, J=6.8 \mathrm{~Hz}), 69.99,69.84$ 68.88, 68.65 (br m), 33.67 (d, $J=34.1 \mathrm{~Hz}), 33.04(\mathrm{~d}, J=34.1 \mathrm{~Hz}), 32.00$, $31.62,31.03,30.92,27.82(\mathrm{~d}, J=6.3 \mathrm{~Hz}), 27.59(\mathrm{~d}, J=10.0 \mathrm{~Hz}), 27.14(\mathrm{~d}$ $J=5.8 \mathrm{~Hz}), 27.08,27.06,27.03,26.94(\mathrm{~d}, J=4.8 \mathrm{~Hz}), 26.84,26.20 .{ }^{31} \mathrm{P}\left\{{ }^{1} \mathrm{H}\right\}$ $\operatorname{NMR}\left(202 \mathrm{MHz}, \mathrm{CDCl}_{3}\right): \delta 24.92$ (s). ${ }^{11} \mathrm{~B}\left\{{ }^{1} \mathrm{H}\right\} \mathrm{NMR}\left(160 \mathrm{MHz}, \mathrm{CDCl}_{3}\right): \delta$ 
Lorsqu'un écrit scientifique issu d'une activité de recherche financée au moins pour moitié par des dotations de l'État, des collectivités territoriales ou des établissements publics, par des subventions d'agences de financement nationales ou par des fonds de l'Union européenne est publié dans un périodique paraissant au moins une fois par an, son auteur dispose, même après avoir accordé des droits exclusifs à un éditeur, du droit de mettre à disposition gratuitement dans un format ouvert, par voie numérique, sous réserve de l'accord des éventuels coauteurs, la version finale de son manuscrit acceptée pour publication, dès lors que l'éditeur met lui-même celle-ci gratuitement à disposition par voie numérique ou, à défaut, à l'expiration d'un délai courant à compter de la date de la première publication. Ce délai est au maximum de six mois pour une publication dans le domaine des sciences, de la technique et de la médecine et de douze mois dans celui des sciences humaines et sociales.

42.41 (s). Anal. Calc. for $\mathrm{C}_{31} \mathrm{H}_{50} \mathrm{BFeO} \mathrm{B}_{2} \mathrm{P} \cdot 0.15$ dichloromethane 0.15 hexane (578.03): C $66.60 \%, \mathrm{H} 9.14 \%$. Found C $66.30 \%, \mathrm{H} 9.67 \%$. ESI+ MS calc. for $\mathrm{C}_{31} \mathrm{H}_{50} \mathrm{BFeNaO} 2 \mathrm{P}\left([\mathrm{M}+\mathrm{Na}]^{+}\right)$: 575.2889 , found: 575.2892 .

Synthesis of 20 . Compound 20 was prepared similarly to 19 , starting with 1-(diisopropylphosphanyl)-1'-bromo-3,3'-di-tert-butylferrocene-borane $(17 ; 200 \mathrm{mg}, 0.39 \mathrm{mmol})$, anhydrous diethyl ether $(8 \mathrm{~mL})$ and $n$-butyllithium ( $0.37 \mathrm{~mL}$ of $1.6 \mathrm{M}$ solution in hexane, $0.59 \mathrm{mmol})$. Yield: $88 \mathrm{mg}(45 \%)$, orange solid. Crystals used for structure determination were obtained from chloroform-hexane.

${ }^{1} \mathrm{H}$ NMR $\left(500 \mathrm{MHz}, \mathrm{CDCl}_{3}\right): \delta 4.90(1 \mathrm{H}, \mathrm{dd}, J=2.6,1.3 \mathrm{~Hz}, \mathrm{Cp}), 4.82(1$ $\mathrm{H}, \mathrm{t}, J=1.5 \mathrm{~Hz}, \mathrm{Cp}), 4.50(1 \mathrm{H}, \mathrm{q}, J=1.9 \mathrm{~Hz}, \mathrm{Cp}), 4.46(1 \mathrm{H}, \mathrm{dd}, J=2.7$ $1.6 \mathrm{~Hz}, \mathrm{Cp}), 4.20(1 \mathrm{H}, \mathrm{d}, J=1.5 \mathrm{~Hz}, \mathrm{Cp}), 4.19(1 \mathrm{H}, \mathrm{dd}, J=2.8,1.5 \mathrm{~Hz}$, Cp), $2.36-2.25(1 \mathrm{H}, \mathrm{d}$ of sept, $J=7.2,10.6 \mathrm{~Hz}, i-\mathrm{Pr}), 2.22-2.11(1 \mathrm{H}, \mathrm{d}$ of sept, $J=7.1,11.7 \mathrm{~Hz}, i-\mathrm{Pr}), 1.30(9 \mathrm{H}, \mathrm{s}, t-\mathrm{Bu}), 1.28(9 \mathrm{H}, \mathrm{s}, t-\mathrm{Bu}), 1.23$ $-1.14(9 \mathrm{H}, \mathrm{m}, i-\mathrm{Pr}), 1.03(3 \mathrm{H}, \mathrm{dd}, J=14.6,7.1 \mathrm{~Hz}, i-\operatorname{Pr}), 0.75-0.36$ (3 $\mathrm{H}$, br m, BH $\left.{ }_{3}\right) .{ }^{13} \mathrm{C}\left\{{ }^{1} \mathrm{H}\right\} \mathrm{NMR}\left(126 \mathrm{MHz}, \mathrm{CDCl}_{3}\right): \delta 177.53$ (s, $\left.\mathrm{COOH}\right)$ 107.82, 107.14 (d, $J=5.4 \mathrm{~Hz}, 74.19(\mathrm{br} \mathrm{m}), 72.42,71.46(\mathrm{~d}, J=55 \mathrm{~Hz})$, $70.54(\mathrm{~d}, J=5.1 \mathrm{~Hz}$ ), 70.11, 69.61, 68.79, 68.34 (br m), 31.98, 31.6, 31.04, 30.96, $24.38(\mathrm{~d}, J=34.9 \mathrm{~Hz}), 23.71(\mathrm{~d}, J=34.0 \mathrm{~Hz}), 18.56,18.12,18.08$ (d, $J=2.4 \mathrm{~Hz}), 17.82 .{ }^{31} \mathrm{P}\left\{{ }^{1} \mathrm{H}\right\} \mathrm{NMR}\left(202 \mathrm{MHz}, \mathrm{CDCl}_{3}\right): \delta 30.96(\mathrm{~s}) .{ }^{11} \mathrm{~B}\left\{{ }^{1} \mathrm{H}\right\}$ NMR (160 MHz, $\left.\mathrm{CDCl}_{3}\right)$ : $\delta-42.92$ (s). IR (ATR): $2960 \mathrm{~m}, 2870 \mathrm{~m}, 2382 \mathrm{~m}$, 1662 vs $(\mathrm{COOH}), 1477 \mathrm{~s}, 1461 \mathrm{~s}, 1385 \mathrm{w}, 1359 \mathrm{~m}, 1302 \mathrm{w}, 1254 \mathrm{~s}, 1177$ m, $1141 \mathrm{w}, 1071 \mathrm{~m}, 1044 \mathrm{~m}, 921 \mathrm{~m}, 877 \mathrm{w}, 854 \mathrm{w}, 832 \mathrm{w}, 746 \mathrm{~m}, 688 \mathrm{~m}$, 659 w, $607 \mathrm{~m} \mathrm{~cm}^{-1}$. Anal. Calc. for $\mathrm{C}_{25} \mathrm{H}_{42} \mathrm{BFeO}_{2} \mathrm{P} \cdot 0.8 \mathrm{H}_{2} \mathrm{O} \cdot 0.5$ hexane (524.35): C $63.49 \%, \mathrm{H} 9.63 \%$. Found C $63.59 \%, \mathrm{H} 9.67 \%$. ESI+ MS calc. for $\mathrm{C}_{25} \mathrm{H}_{42} \mathrm{BFeNaO} 2 \mathrm{P}\left([\mathrm{M}+\mathrm{Na}]^{+}\right)$: 495.2263, found: 495.2239 .

Synthesis of 22 from 21 . Compound $21^{[12]}$ (50 mg, $0.1 \mathrm{mmol},{ }^{31} \mathrm{P} \delta-$ 17.77) dissolved in dichloromethane $(2 \mathrm{~mL})$ was cooled at $0^{\circ} \mathrm{C}$. Then, $\mathrm{BH}_{3} \cdot \mathrm{SMe}_{2}(0.08 \mathrm{~mL}$ of $2.0 \mathrm{M}$ solution in THF, $0.15 \mathrm{mmol}$ ) was added and the mixture was stirred for $2 \mathrm{~h}$ at room temperature before quenching with distilled water. The mixture was partitioned between dichloromethane and brine. The organic layer was separated, washed with brine, dried over anhydrous magnesium sulfate and, finally, evaporated to give compound 22 as a yellow oil. Yield: $35 \mathrm{mg}(67 \%)$

${ }^{1} \mathrm{H}$ NMR $\left(500 \mathrm{MHz}, \mathrm{CDCl}_{3}\right): \delta 7.65(2 \mathrm{H}, \mathrm{ddd}, \mathrm{J}=11.0,8.3,1.5 \mathrm{~Hz}, \mathrm{Ph})$, $7.49-7.32(8 \mathrm{H}, \mathrm{m}, \mathrm{Ph}), 4.40(1 \mathrm{H}, \mathrm{dt}, J=2.4,1.2 \mathrm{~Hz}, \mathrm{Cp}), 4.30(1 \mathrm{H}$, dd, $J=2.4,1.3 \mathrm{~Hz}, \mathrm{Cp}), 4.26-4.20\left(4 \mathrm{H}, \mathrm{m}, \mathrm{Cp}\right.$ and $\left.\mathrm{CH}_{2} \mathrm{OH}\right), 4.13(1 \mathrm{H}, \mathrm{t}, J$ $=1.5 \mathrm{~Hz}, \mathrm{Cp}), 4.01(1 \mathrm{H}, \mathrm{dd}, J=2.5,1.6 \mathrm{~Hz}, \mathrm{Cp}), 1.87(1 \mathrm{H}, \mathrm{s}, \mathrm{OH}), 1.29$ $(9 \mathrm{H}, \mathrm{s}, t-\mathrm{Bu}), 0.86(10 \mathrm{H}, \mathrm{s}, t-\mathrm{Bu}) \cdot{ }^{13} \mathrm{C}\left\{{ }^{1} \mathrm{H}\right\} \mathrm{NMR}\left(126 \mathrm{MHz}, \mathrm{CDCl}_{3}\right): \delta 133.5$ (d, $J=9.6 \mathrm{~Hz}), 132.51(\mathrm{~d}, J=9.5 \mathrm{~Hz}), 132.554(\mathrm{~d}, J=59.2 \mathrm{~Hz}), 131.25$ (d, $J=2.5 \mathrm{~Hz}), 130.82(\mathrm{~d}, J=2.4 \mathrm{~Hz}), 130.67(\mathrm{~d}, J=59.3 \mathrm{~Hz}), 128.76(\mathrm{~d}, J=$ $10.0 \mathrm{~Hz}$ ), 128.57 (d, $J=10.2 \mathrm{~Hz}), 106.12(\mathrm{~d}, J=7.3 \mathrm{~Hz}), 103.13,88.59$, $72.5(\mathrm{~d}, J=6.0 \mathrm{~Hz}), 70.23(\mathrm{~d}, J=12.3 \mathrm{~Hz}), 69.47,69.06(\mathrm{~d}, J=6.6 \mathrm{~Hz})$, $68.59(\mathrm{~d}, J=68.5 \mathrm{~Hz}), 67.12,66.20,60.33,31.56,31.31,31.11,30.33$. ${ }^{31} \mathrm{P}\left\{{ }^{1} \mathrm{H}\right\}$ NMR $\left(202 \mathrm{MHz}, \mathrm{CDCl}_{3}\right): \delta 15.96\left(\mathrm{~d},{ }^{1} \mathrm{~J}_{\mathrm{BP}}=51.6 \mathrm{~Hz}\right) .{ }^{11} \mathrm{~B}\left\{{ }^{1} \mathrm{H}\right\} \mathrm{NMR}$ $\left(160 \mathrm{MHz}, \mathrm{CDCl}_{3}\right): \delta-38.46$ (s). This oily product did not furnish satisfactory EA. ESI+ MS calc. for $\mathrm{C}_{31} \mathrm{H}_{40} \mathrm{BFeNaOP}\left([\mathrm{M}+\mathrm{Na}]^{+}\right)$: 549.2157 , found: 549.2148 .

Synthesis of 23. Adduct $15(67 \mathrm{mg}, 0.12 \mathrm{mmol})$ was dissolved in anhydrous THF $(2 \mathrm{~mL})$ in an oven-dried reaction flask. The stirring solution was cooled to approximately $-80^{\circ} \mathrm{C}$ and treated with $n$-butyllithium $(0.1 \mathrm{~mL}$ of $1.6 \mathrm{M}$ in hexane, $0.16 \mathrm{mmol})$. After stirring for $20 \mathrm{~min}$, neat DMF $(0.05$ $\mathrm{mL}, 0.65 \mathrm{mmol}$ ) was added and the mixture was stirred for $30 \mathrm{~min}$ at $80^{\circ} \mathrm{C}$. The mixture was allowed to warm to room temperature during 90 min before quenching with brine $(2 \mathrm{~mL})$ and $3 \mathrm{M} \mathrm{HCl}(2 \mathrm{~mL})$. Subsequently, the mixture was extracted with ethyl acetate and washed with brine, dried over $\mathrm{MgSO}_{4}$ and evaporated. The crude product was purified by chromatography over a silica gel column using pentane-ethyl acetate
(19:1) as the eluent. The two first minor bands were discarded and the following major one collected and evaporated to give compound $\mathbf{2 3}$ as a red solid. Yield: $24.3 \mathrm{mg}(39 \%)$.

${ }^{1} \mathrm{H}$ NMR $\left(500 \mathrm{MHz}, \mathrm{CDCl}_{3}\right): \delta 9.33(1 \mathrm{H}, \mathrm{s}, \mathrm{CHO}), 7.63-7.33(10 \mathrm{H}, \mathrm{m}$, $\mathrm{Ph}), 4.79(1 \mathrm{H}, \mathrm{dd}, J=2.8,1.3 \mathrm{~Hz}, \mathrm{Cp}), 4.70(1 \mathrm{H}, \mathrm{t}, J=1.5 \mathrm{~Hz}, \mathrm{Cp}), 4.45$ $(2 \mathrm{H}, \mathrm{p}, J=2.0 \mathrm{~Hz}, \mathrm{Cp}), 4.33(1 \mathrm{H}, \mathrm{dt}, J=2.8,1.4 \mathrm{~Hz}, \mathrm{Cp}), 4.21(1 \mathrm{H}, \mathrm{q}, J$ $=1.6 \mathrm{~Hz}, \mathrm{Cp}), 1.28(9 \mathrm{H}, \mathrm{s}, t-\mathrm{Bu}), 1.04(9 \mathrm{H}, \mathrm{s}, t-\mathrm{Bu}) \cdot{ }^{13} \mathrm{C}\left\{{ }^{1} \mathrm{H}\right\} \mathrm{NMR}(126$ $\left.\mathrm{MHz}, \mathrm{CDCl}_{3}\right): \delta 195.38$ (s, CHO), $133.00(\mathrm{~d}, J=4.3 \mathrm{~Hz}), 132.92(\mathrm{~d}, J=4.5$ $\mathrm{Hz}), 131.46(\mathrm{~d}, J=15.7 \mathrm{~Hz}), 131.40(\mathrm{~d}, J=3.9 \mathrm{~Hz}), 131.14(\mathrm{~d}, J=2.3 \mathrm{~Hz})$, $130.99(\mathrm{~d}, J=17.4 \mathrm{~Hz}), 128.86(\mathrm{~d}, J=10.2 \mathrm{~Hz}), 128.75(\mathrm{~d}, J=10.0 \mathrm{~Hz})$, 108.37, $108.31(\mathrm{~d}, J=6.2 \mathrm{~Hz}), 78.46,74.53(\mathrm{~d}, J=9.7 \mathrm{~Hz}), 71.33(\mathrm{~d}, J=$ $8.1 \mathrm{~Hz}), 71.05,70.42(\mathrm{~d}, J=65.5 \mathrm{~Hz}), 69.75,69.57,68.57(\mathrm{~d}, J=7.2 \mathrm{~Hz})$, 31.48, 31.02, 30.70. ${ }^{31} \mathrm{P}\left\{{ }^{1} \mathrm{H}\right\} \mathrm{NMR}\left(202.38 \mathrm{MHz}, \mathrm{CDCl}_{3}\right): \delta 15.84\left(\mathrm{~d},{ }^{1} \mathrm{~J} \mathrm{BP}=\right.$ $47.6 \mathrm{~Hz}) .{ }^{11} \mathrm{~B}\left\{{ }^{1} \mathrm{H}\right\} \mathrm{NMR}\left(160.39 \mathrm{MHz}, \mathrm{CDCl}_{3}\right): \delta-38.25$ (s). Anal. Calc. for $\mathrm{C}_{31} \mathrm{H}_{38} \mathrm{BFeOP} \cdot 0.7 \mathrm{H}_{2} \mathrm{O} \cdot 0.9$ pentane (601.84): $\mathrm{C} 70.85 \%, \mathrm{H} 8.41 \%$. Found C $70.73 \%, \mathrm{H} 8.49 \%$. ESI+ MS calc. for $\mathrm{C}_{31} \mathrm{H}_{38} \mathrm{BFeNaOP}\left([\mathrm{M}+\mathrm{Na}]^{+}\right)$: 547.2000 , found: 547.1988 .

Synthesis of 24. Compound 24 was prepared similarly to 23 , starting with 1-(dicyclohexylphosphanyl)-1'-bromo-3,3'-di-tert-butylferrocene-borane (16; $100 \mathrm{mg}, 0.17 \mathrm{mmol})$, anhydrous THF (5 mL), $n$-Butyllithium $(0.25 \mathrm{~mL}$ of $1.6 \mathrm{M}$ solution in hexane, $0.4 \mathrm{mmol})$ and anhydrous DMF $(0.11 \mathrm{~mL}, 1.43$ $\mathrm{mmol})$. The crude product was purified by chromatography over a silica gel column using pentane-ethyl acetate (9:1). Yield: $61.8 \mathrm{mg}(67 \%)$, red solid. Crystals used for structure determination were obtained from hot heptane.

${ }^{1} \mathrm{H}$ NMR $\left(600 \mathrm{MHz}, \mathrm{CDCl}_{3}\right): \delta 10.08(1 \mathrm{H}, \mathrm{s}, \mathrm{CHO}), 4.86(1 \mathrm{H}, \mathrm{br} \mathrm{s}, \mathrm{Cp})$, $4.81(1 \mathrm{H}, \mathrm{s}, \mathrm{Cp}), 4.54(1 \mathrm{H}, \mathrm{br} \mathrm{s}, \mathrm{Cp}), 4.39(1 \mathrm{H}, \mathrm{br} \mathrm{s}, \mathrm{Cp}), 4.20(1 \mathrm{H}, \mathrm{br} \mathrm{s}$ Cp), 4.17 (1 H, br s, Cp), $1.93-1.75(10 \mathrm{H}, \mathrm{m}, \mathrm{Cy}), 1.73-1.67(2 \mathrm{H}, \mathrm{m}$, Сy), $1.40-1.30(4 \mathrm{H}, \mathrm{m}, \mathrm{Cy}), 1.28(9 \mathrm{H}, \mathrm{s}, t-\mathrm{Bu}), 1.27(9 \mathrm{H}, \mathrm{s}, t-\mathrm{Bu}), 1.25$ $-1.10(6 \mathrm{H}, \mathrm{m}, \mathrm{Cy}), 0.97-0.17\left(3 \mathrm{H}, \mathrm{br} \mathrm{m}, \mathrm{BH}_{3}\right) .{ }^{13} \mathrm{C}\left\{{ }^{1} \mathrm{H}\right\} \mathrm{NMR}(151 \mathrm{MHz}$, $\left.\mathrm{CDCl}_{3}\right): \delta 195.61$ (s, CHO), 108.53, $106.78(\mathrm{~d}, J=5.5 \mathrm{~Hz}), 78.58,73.59$ (d, $J=6.2 \mathrm{~Hz}), 71.28(\mathrm{~d}, J=53.2 \mathrm{~Hz}), 71.02,70.20,70.17(\mathrm{~d}, J=6.3 \mathrm{~Hz})$ $69.11,67.58(\mathrm{~d}, J=6.3 \mathrm{~Hz}), 33.29(\mathrm{~d}, J=34.0 \mathrm{~Hz}), 33.27(\mathrm{~d}, J=33.8 \mathrm{~Hz})$, $31.93,31.53,31.06,30.91,28.02,27.93,27.85,27.72,27.13$ (d, $J=4.4$ $\mathrm{Hz}), 27.05(\mathrm{~d}, J=2.2 \mathrm{~Hz}), 26.97(\mathrm{~d}, J=3.3 \mathrm{~Hz}), 26.89,26.18,26.13$. ${ }^{31} \mathrm{P}\left\{{ }^{1} \mathrm{H}\right\}$ NMR $\left(243 \mathrm{MHz}, \mathrm{CDCl}_{3}\right): \delta 24.43(\mathrm{~d}, J=62.3 \mathrm{~Hz}) .{ }^{11} \mathrm{~B}\left\{{ }^{1} \mathrm{H}\right\} \mathrm{NMR}$ $\left(193 \mathrm{MHz}, \mathrm{CDCl}_{3}\right.$ ): $\delta-42.07$ (br s). Anal. Calc. for $\mathrm{C}_{31} \mathrm{H}_{50} \mathrm{BFeOP} \cdot 0.8 \mathrm{H}_{2} \mathrm{O}$ $\cdot 0.3$ heptane (580.67): C $68.47 \%$, H 9.79\%. Found C $68.44 \%, \mathrm{H} 9.82 \%$. $\mathrm{ESI}+\mathrm{MS}$ calc. for $\mathrm{C}_{31} \mathrm{H}_{50} \mathrm{BFeNaOP}\left([\mathrm{M}+\mathrm{Na}]^{+}\right): 559.2939$, found: 559.2926 .

Synthesis of 25 . Compound 25 was prepared similarly to 23 , starting with 1-(dicyclohexylphosphanyl)-1'-bromo-3,3'-di-tert-butylferrocene-borane (17; $200 \mathrm{mg}, 0.39 \mathrm{mmol})$, anhydrous THF (4 mL), $n$-Butyllithium $(0.29 \mathrm{~mL}$ of $1.6 \mathrm{M}$ solution in hexane, $0.47 \mathrm{mmol})$ and anhydrous DMF $(0.155 \mathrm{~mL}$, $1.95 \mathrm{mmol})$. Yield: $107.1 \mathrm{mg}(60 \%)$, red solid. Crystals used for structure determination were obtained from hot heptane.

${ }^{1} \mathrm{H}$ NMR $\left(600 \mathrm{MHz}, \mathrm{CDCl}_{3}\right)$ : $\delta 10.07(1 \mathrm{H}, \mathrm{s}, \mathrm{CHO}), 4.86(1 \mathrm{H}, \mathrm{br} \mathrm{s}, \mathrm{Cp})$, $4.83(1 \mathrm{H}, \mathrm{s}, \mathrm{Cp}), 4.55(1 \mathrm{H}, \mathrm{br} \mathrm{s}, \mathrm{Cp}), 4.45(1 \mathrm{H}, \mathrm{br} \mathrm{s}, \mathrm{Cp}), 4.22(1 \mathrm{H}, \mathrm{br} \mathrm{s}$, Cp), $4.21(1 \mathrm{H}, \mathrm{br} \mathrm{s}, \mathrm{Cp}), 2.20-2.09(2 \mathrm{H}, \mathrm{m}, i-\mathrm{Pr}), 1.28(9 \mathrm{H}, \mathrm{s}, t-\mathrm{Bu}), 1.26$ $(9 \mathrm{H}, \mathrm{s}, t-\mathrm{Bu}), 1.22-1.13(9 \mathrm{H}, \mathrm{m}, i-\mathrm{Pr}), 1.09-1.03(3 \mathrm{H}, \mathrm{dd}, J=7.0,14.6$ $\mathrm{Hz}, i-\operatorname{Pr}), 0.95-0.23\left(3 \mathrm{H}, \mathrm{br} \mathrm{m}, \mathrm{BH}_{3}\right) .{ }^{13} \mathrm{C}\left\{{ }^{1} \mathrm{H}\right\} \mathrm{NMR}\left(151 \mathrm{MHz}, \mathrm{CDCl}_{3}\right): \delta$ 195.51 (s, CHO), 108.69, 106.94 (d, $J=5.4 \mathrm{~Hz}$ ), 78.52, 73.86 (d, $J=8.2$ $\mathrm{Hz}), 71.24(\mathrm{~d}, J=52.6 \mathrm{~Hz}), 71.09,70.51,69.89(\mathrm{~d}, J=5.4 \mathrm{~Hz}), 68.78$, $67.67(\mathrm{~d}, J=6.2 \mathrm{~Hz}), 31.88,31.52,31.07,30.89,23.89(\mathrm{~d}, J=34.3 \mathrm{~Hz})$, $23.56(\mathrm{~d}, J=34.3 \mathrm{~Hz}), 18.49,18.12,18.01,17.83 .{ }^{31} \mathrm{P}\left\{{ }^{1} \mathrm{H}\right\} \mathrm{NMR}(243 \mathrm{MHz}$, $\left.\mathrm{CDCl}_{3}\right): \delta 31.13\left(\mathrm{~d},{ }^{1} \mathrm{JBP}_{\mathrm{BP}}=78.9 \mathrm{~Hz}\right) .{ }^{11} \mathrm{~B}\left\{{ }^{1} \mathrm{H}\right\} \mathrm{NMR}\left(193 \mathrm{MHz}, \mathrm{CDCl}_{3}\right): \delta$ $-41.66--43.46$ (m). Anal. Calc. for $\mathrm{C}_{25} \mathrm{H}_{42} \mathrm{BFeOP}$ (456.24): $\mathrm{C} 65.82 \%, \mathrm{H}$ $9.28 \%$. Found C $65.56 \%, \mathrm{H} 9.41 \%$. ESI+ MS calc. for $\mathrm{C}_{25} \mathrm{H}_{42} \mathrm{BFeNaOP}$ ([M $\left.+\mathrm{Na}^{+}\right): 479.2313$, found: 479.2315 . 
Lorsqu'un écrit scientifique issu d'une activité de recherche financée au moins pour moitié par des dotations de l'État, des collectivités territoriales ou des établissements publics, par des subventions d'agences de financement nationales ou par des fonds de l'Union européenne est publié dans un périodique paraissant au moins une fois par an, son auteur dispose, même après avoir accordé des droits exclusifs à un éditeur, du droit de mettre à disposition gratuitement dans un format ouvert, par voie numérique, sous réserve de l'accord des éventuels coauteurs, la version finale de son manuscrit acceptée pour publication, dès lors que l'éditeur met lui-même celle-ci gratuitement à disposition par voie numérique ou, à défaut, à l'expiration d'un délai courant à compter de la date de la première publication. Ce délai est au maximum de six mois pour une publication dans le domaine des sciences, de la technique et de la médecine et de douze mois dans celui des sciences humaines et sociales.

Synthesis of 26. A thin glass tube was charged under air with 1'(diphenylphosphanyl)-3,3'-di-tert-butylferrocene-1-carboxylic acid (10 $26.3 \mathrm{mg}, 0.05 \mathrm{mmol}$ ) and chloro(dimethylsulfide)gold(I) (14.7 mg, 0.05 $\mathrm{mmol}$ ) in $0.5 \mathrm{~mL}$ of dichloromethane- $\mathrm{D}_{2}$. The mixture was stirring for a few minutes at room temperature to form gold complex $\mathbf{2 6}$ in quantitative yield as testified multinuclear NMR monitoring. Crystal used for structure determination was obtained from $\mathrm{CH}_{2} \mathrm{Cl}_{2}$ - heptane.

${ }^{1} \mathrm{H}$ NMR $\left(500 \mathrm{MHz}, \mathrm{CD}_{2} \mathrm{Cl}_{2}\right): \delta 7.81-7.74(2 \mathrm{H}, \mathrm{m}, \mathrm{Ph}), 7.60-7.49(3 \mathrm{H}$, m, Ph), $7.46-7.30(5 \mathrm{H}, \mathrm{m}, \mathrm{Ph}), 4.88(1 \mathrm{H}, \mathrm{dd}, J=2.7,1.3 \mathrm{~Hz}, \mathrm{Cp}), 4.55$ (1 H, t, J = $1.5 \mathrm{~Hz}, \mathrm{Cp}), 4.49-4.41(2 \mathrm{H}, \mathrm{m}, \mathrm{Cp}), 4.37$ (1 H, dd, $J=2.7$, $1.6 \mathrm{~Hz}, \mathrm{Cp}), 4.27(1 \mathrm{H}, \mathrm{td}, J=2.5,1.4 \mathrm{~Hz}, \mathrm{Cp}), 1.34(9 \mathrm{H}, \mathrm{s}, t-\mathrm{Bu}), 0.93$ (9 $\mathrm{H}, \mathrm{s}, t-\mathrm{Bu}) .{ }^{13} \mathrm{C}\left\{{ }^{1} \mathrm{H}\right\} \mathrm{NMR}\left(126 \mathrm{MHz}, \mathrm{CD}_{2} \mathrm{Cl}_{2}\right): \delta 174.32(\mathrm{~s}, \mathrm{COOH}), 135.08$ (d, $J=14.3 \mathrm{~Hz}$ ), 133.26 (d, $J=13.5 \mathrm{~Hz}), 132.47$ (d, $J=2.6 \mathrm{~Hz}), 132.02$ (d, $J=63.0 \mathrm{~Hz}), 131.60(\mathrm{~d}, J=2.6 \mathrm{~Hz}), 130.82(\mathrm{~d}, J=65.0 \mathrm{~Hz}), 129.55$ (d, J $=12.0 \mathrm{~Hz}), 129.22(\mathrm{~d}, J=11.6 \mathrm{~Hz}), 108.64(\mathrm{~d}, J=9.1 \mathrm{~Hz}), 107.65,74.80$ (d, $J=18.8 \mathrm{~Hz}), 73.18,72.56(\mathrm{~d}, J=7.7 \mathrm{~Hz}), 71.03,70.48(\mathrm{~d}, J=71.5 \mathrm{~Hz})$, 70.27, 69.46, 31.58, 31.38, 31.35, 30.65. ${ }^{31} \mathrm{P}\left\{{ }^{1} \mathrm{H}\right\}$ NMR $\left(202 \mathrm{MHz}, \mathrm{CD}_{2} \mathrm{Cl}_{2}\right)$ : $\delta 26.48$ (s). Anal. Calc. for $\mathrm{C}_{31} \mathrm{H}_{35} \mathrm{AuClFeO}_{2} \mathrm{P}$ (758.85): C 49.07\%, H $4.65 \%$. Found C 49.25\%, H 4.65\%. ESI+ MS calc. for $\mathrm{C}_{31} \mathrm{H}_{35} \mathrm{AuClFeNaO} 2 \mathrm{P}\left([\mathrm{M}+\mathrm{Na}]^{+}\right): 781.0976$, found: 781.0979 .

Synthesis of $\mathbf{2 7}$. Compound $\mathbf{2 7}$ was prepared similarly to $\mathbf{2 6}$, starting with 20 (25.5 mg, $0.05 \mathrm{mmol}$ ), chloro(dimethylsulfide)gold(I) (14.7 mg, 0.05 $\mathrm{mmol})$ and dichloromethane- $\mathrm{D}_{2}(0.5 \mathrm{~mL})$ to form gold complex 26 in quantitative yield as evidenced by ${ }^{1} \mathrm{H}$ and ${ }^{31} \mathrm{P}$ NMR analysis.

${ }^{1} \mathrm{H}$ NMR $\left(500 \mathrm{MHz}, \mathrm{CD}_{2} \mathrm{Cl}_{2}\right): \delta 9.54(1 \mathrm{H}, \mathrm{s}, \mathrm{CHO}), 7.63-7.50(6 \mathrm{H}, \mathrm{m}$, $\mathrm{Ph}), 7.49$ - 7.44 (4 H, m, Ph), 4.80 (1 H, dd, J=2.8, $1.3 \mathrm{~Hz}, \mathrm{Cp}), 4.64$ (1 $\mathrm{H}, \mathrm{t}, J=1.4 \mathrm{~Hz}, \mathrm{Cp}), 4.51(1 \mathrm{H}, \mathrm{t}, J=2.2 \mathrm{~Hz}, \mathrm{Cp}), 4.46(1 \mathrm{H}, \mathrm{dt}, J=2.6$, 1.4.6 Hz, Cp), 4.43 (1 H, td, J = 2.8, 1.4 Hz, Cp), 4.28 (1 H, dt, J = 3.1, 1.5 $\mathrm{Hz}, \mathrm{Cp}), 1.28(9 \mathrm{H}, \mathrm{s}, t-\mathrm{Bu}), 1.07(9 \mathrm{H}, \mathrm{s}, t-\mathrm{Bu}) \cdot{ }^{13} \mathrm{C}\left\{{ }^{1} \mathrm{H}\right\} \mathrm{NMR}(126 \mathrm{MHz}$, $\mathrm{CD}_{2} \mathrm{Cl}_{2}$ ): $\delta 194.22$ (s, COOH), 134.31 (d, $\left.J=13.9 \mathrm{~Hz}\right), 133.99$ (d, J=14.0 $\mathrm{Hz}), 132.23(\mathrm{~d}, J=2.7 \mathrm{~Hz}), 132.17(\mathrm{~d}, J=2.7 \mathrm{~Hz}), 131.66(\mathrm{~d}, J=55.2 \mathrm{~Hz})$, $131.15(\mathrm{~d}, J=55.5 \mathrm{~Hz}), 129.55(\mathrm{~d}, J=2.0 \mathrm{~Hz}), 129.45(\mathrm{~d}, J=1.9 \mathrm{~Hz})$, $109.16(\mathrm{~d}, J=7.7 \mathrm{~Hz}), 108.92,78.95,75.22(\mathrm{~d}, J=12.5 \mathrm{~Hz}), 72.16(\mathrm{~d}, J=$ $13.2 \mathrm{~Hz}), 71.54,70.57$ (d, $J=70.9 \mathrm{~Hz}), 70.24,69.88,69.83(\mathrm{~d}, J=8.1 \mathrm{~Hz})$, 31.63, 31.38, 31.23, 30.86. ${ }^{31} \mathrm{P}\left\{{ }^{1} \mathrm{H}\right\}$ NMR (202 MHz, $\left.\mathrm{CD}_{2} \mathrm{Cl}_{2}\right): \delta 28.30$ (s). Anal. Calc. for $\mathrm{C}_{31} \mathrm{H}_{35} \mathrm{AuClFeOP} \cdot 0.8 \mathrm{H}_{2} \mathrm{O}$ (757.27): C 49.38\%, H 4.89\%. Found C $49.28 \%$, H 5.02\%. ESI+ MS calc. for $\mathrm{C}_{31} \mathrm{H}_{35} \mathrm{AuClFeNaOP}$ ([M + $\left.\mathrm{Na}]^{+}\right):$765.1027, found: 765.1023

X-Ray crystallography. Full-sphere diffraction data for $\mathbf{1 0}$ and $\mathbf{1 8}$ were collected with a Bruker D8 VENTURE Kappa Duo PHOTON100 instrument equipped with a I $\mu \mathrm{S}$ micro-focus $\mathrm{X}$-ray tube. The data for other compounds 10Se, 17, 24, 26 and 27 were recorded with a Bruker D8 Venture diffractometer and the data for $\mathbf{2 0}$ and 25, on a Bruker CCD diffractometer, All the diffractometers were equipped with Cryostream Cooler (Oxford Cryosystems) and Mo K $\alpha$ radiation was used in all cases.

The structures were solved by direct methods (SHELXT) ${ }^{[21]}$ and then refined using a full-matrix least squares routine based on $F^{2}$ with $S H E L X L$ 2014/2018.[22] All non-hydrogen atoms were refined with anisotropic displacement parameters. $\mathrm{OH}$ and $\mathrm{BH}$ hydrogens were identified on the difference electron density maps and were refined as riding atoms wit $U_{\text {iso }}(\mathrm{H})$ set to a multiple of $U_{\text {eq }}$ of the bonding atom. Hydrogens residing on carbon atoms were included in their theoretical positions and refined similarly. Relevant crystallographic data, data collection and structure refinement parameters are presented in Supporting Information. CCDC 1877094-1877102 (10, 10Se, 17, 18, 24, 25, 26 and 27 respectively) contains the supplementary crystallographic data for this paper. These data can be obtained free of charge from The Cambridge Crystallographic Data Centre via www.ccdc.cam.ac.uk/data request/cif.

\section{Acknowledgments}

This work was supported by the ANR-PRC 2016 program (ALCATRAS, ANR-16-CE07-0001-01), the CNRS, Université de Bourgogne, Conseil Régional de Bourgogne through the plan d'actions régional pour l'innovation (PARI) and the fonds européen de développement regional (FEDER), and by the Charles University Research Centre program No. UNCE/SCI/014.

Keywords: Ferrocenyl phosphanes - Brønsted acids - Hybrid ligands $\bullet$ Coordination compounds $\cdot$ Structures

\section{References}

[1] a) P. Štěpnička in Ferrocenes: Ligands, Materials and Biomolecules John Wiley \& Sons, Chichester, 2008; b) K. S. Gan, T. S. A. Hor, Ferrocenes: Homogeneous Catalysis, Organic Synthesis, Material Science (Eds.: A. Togni, T. Hayashi), VCH Verlagsgesellschaft, Weinheim, Germany 1995 ; c) G. Jaouen, N. Metzler-Nolte (Eds.), Topics in Organometallic Chemistry, vol. 32: Medicinal Organometallic Chemistry, Springer, Berlin, 2010.

[2] a) A. Fihri, P. Meunier, J.-C. Hierso, Coord. Chem. Rev. 2007, 251, 2017-2055; b) L.-X. Dai, X.-L. Hou (Eds.), Chiral Ferrocenes in Asymmetric Catalysis: Synthesis and Applications, Wiley- $\mathrm{VCH}$, Weinheim, Germany, 2010.

[3] P. Štěpnička, Chem. Soc. Rev. 2012, 41, 4273-4305.

[4] N. Dwadnia, J. Roger, N. Pirio, H. Cattey, J.-C. Hierso, Coord. Chem. Rev. 2018, 355, 74-100

[5] a) J. Podlaha, P. Štěpnička, J. Ludvik, I. Císařová, Organometallics 1996 15, 543-550; b) P. Štěpnička, Eur. J. Inorg. Chem. 2005, 3787-3803; c) J. Schulz, P. Vosáhlo, F. Uhlík, I. Císařová, P. Štěpnička, Organometallics 2017, 36, 1828-1841.

[6] P. Štěpnička, Coord. Chem. Rev. 2017, 353, 223-246.

[7] J. Schulz, I. Císařová, P. Štěpnička, Organometallics 2012, 31, 729-738; b) J. Schulz, F. Horký, I. Císařová, P. Štěpnička, Catalysts 2017, 7, art $n^{\circ} 167$.

[8] a) Representative examples: a) O. Bárta, I. Císařová, P. Štěpnička, J. Organomet. Chem. 2018, 855, 26-32; b) M. Zábranský, A. Machara, I. Císařová, P. Štěpnička, Eur. J. Inorg. Chem. 2017, 4850-4860; c) P. Štěpnička, M. Zábranský, I. Císařová, J. Organomet. Chem. 2017, 846, 193-200; d) M. Zábranský, I. Císařová, P. Štěpnička, Eur. J. Inorg. Chem. 2017, 2557-2572; e) H. Charvátová, I. Císařová, P. Štěpnička, Eur. J. Inorg. Chem. 2017, 288-296; f) O. Bárta, I. Císařová, P. Štěpnička, Eur J. Inorg. Chem. 2017, 489-495; g) K. Škoch, F. Uhlik, I. Císařová, P. Štěpnička, Dalton Trans. 2016, 45, 10655-10671; h) K. Škoch, I. Císařová, P. Štěpnička, Chem. Eur. J. 2015, 21, 15998-16004, i) P Štěpnička, M. Zábranský, I. Císařová, ChemistryOpen 2012, 1, 71-79.

[9] a) N. Dwadnia, F. Allouch, N. Pirio, J. Roger, H. Cattey, S. Fournier, M. J. Penouilh, C. H. Devillers, D. Lucas, D. Naoufal, R. Ben Salem, J.-C. Hierso, Organometallics 2013, 32, 5784-5797; b) F. Allouch, N. Dwadnia, N.V. Vologdin, Y.V. Svyaschenko, H. Cattey, M.-J. Penouilh, J. Roger, D. Naoufal, R. Ben Salem, N. Pirio, J.-C. Hierso, Organometallics 2015 34, 5015-5028; c) J.-C. Hierso, R. V. Smaliy, R. Amardeil, P. Meunier, Chem. Soc. Rev. 2007, 36, 1754-1769; d) J.-C. Hierso, M. Beaupérin, P. Meunier, Eur. J. Inorg. Chem. 2007, 3767-3780.

[10] a) M. Beaupérin, R. Smaliy, H. Cattey, P. Meunier, J. Ou, P. H. Toy, J.C. Hierso, Chem. Commun. 2014, 50, 9505-9508; b) M. Beaupérin, R. 
Lorsqu'un écrit scientifique issu d'une activité de recherche financée au moins pour moitié par des dotations de l'État, des collectivités territoriales ou des établissements publics, par des subventions d'agences de financement nationales ou par des fonds de l'Union européenne est publié dans un périodique paraissant au moins une fois par an, son auteur dispose, même après avoir accordé des droits exclusifs à un éditeur, du droit de mettre à disposition gratuitement dans un format ouvert, par voie numérique, sous réserve de l'accord des éventuels coauteurs, la version finale de son manuscrit acceptée pour publication, dès lors que l'éditeur met lui-même celle-ci gratuitement à disposition par voie numérique ou, à défaut, à l'expiration d'un délai courant à compter de la date de la première publication. Ce délai est au maximum de six mois pour une publication dans le domaine des sciences, de la technique et de la médecine et de douze mois dans celui des sciences humaines et sociales.

Smaliy, H. Cattey, P. Meunier, J. Ou, P. H. Toy, J.-C. Hierso, ChemPlusChem, 2015, 1, 119-129.

[11] a) For gold-catalysed Suzuki cross-coupling, see: N. Dwadnia, J. Roger, N. Pirio, H. Cattey, R. Ben Salem, J.-C. Hierso, Chem. Asian J. 2017, 12, 459-464; For palladium-catalysed C-H functionalization, see: b) D. Roy, S. Mom, M. Beaupérin, H. Doucet, J.-C. Hierso, Angew. Chem. Int. Ed 2010, 49, 6650-6654; Angew. Chem. 2010, 122, 6800-6804; c) D. Roy, S. Mom, D. Lucas, H. Cattey, J.-C. Hierso, H. Doucet, Chem. Eur. J. 2011, 17, 6453-6461; d) D. Roy, S. Mom, S. Royer, D. Lucas, J.-C. Hierso, H. Doucet, ACS Catal. 2012, 2, 1033-1041; e) X. Wu, J. W. T. See, K. Xu, H. Hirao, J. Roger, J.-C. Hierso, J. Zhou, Angew. Chem. Int. Ed. 2014, 53, 13573-13577; Angew. Chem. 2014, 126, 13791-13795; f) For C-O etherification reactions, see: M. Platon, L. Cui, S. Mom, P. Richard, M. Saeys, J.-C. Hierso, Adv. Synth. Catal. 2011, 353, 34033414 ; g) For C-S thioetherification reactions, see: M. Platon, N. Wijaya, V. Rampazzi, L. Cui, Y. Rousselin, M. Saeys, J.-C. Hierso, Chem. Eur. J. 2014, 20, 12584-12594.

[12] a) E. Lerayer, P. Renaut, J. Roger; N. Pirio, H. Cattey, C. H. Devillers, D. Lucas, J.-C. Hierso, Chem. Commun. 2017, 53, 6017-6020; b) G. Masson, P. Beyer, P. W. Cyr, A. J. Lough, I. Manners, Macromolecules 2006, 39, 3720-3730; c) M. Roemer, D. Heinrich, Y. K. Kang, Y. K. Chung, D. Lentz, Organometallics 2012, 31, 1500-1510.

[13] E. Lerayer, P. Renaut, S. Brandès, H. Cattey, P. Fleurat-Lessard, G. Bouhadir, D. Bourisson, J.-C. Hierso, Inorg. Chem. 2017, 56, 1966-1973.

[14] a) J. Roger, S. Royer, H. Cattey, A. Savateev, R. V. Smaliy, A. N. Kostyuk, J.-C. Hierso, Eur. J. Inorg. Chem. 2017, 330-339; b) V. Rampazzi, J.
Roger, R. Amardeil, M.-J. Penouilh, P. Richard, P. Fleurat-Lessard, J.-C. Hierso, Inorg. Chem. 2016, 55, 10907-10921; c) R. A. Olofson, C. M. Dougherty, J. Am. Chem. Soc. 1973, 95, 977-584; d) W. McFarlane, D. S. Rycroft, J. Chem. Soc., Dalton Trans. 1973, 2162-2166; e) R. D. Kroshefsky, R. Weiss, J. G. Verkade, Inorg. Chem. 1979, 18, 469-472; f) D. W. Allen, B. F. Taylor, J. Chem. Soc., Dalton Trans. 1982, 51-54; g) M. N. Chevykalova, L. F. Manzhukova, N. V. Artemova, Y. N. Luzikov, I. E. Nifantev, E. E. Nifantev, Russ. Chem. Bull. 2003, 52, 78-84.

[15] a) D. Seyferth, H. P. Withers Jr, J. Organomet. Chem. 1980, 185, C1-C5; b) D. Seyferth, H. P. Withers, Jr., Organometallics 1982, 1, 1275-1282.

[16] A. G. Osborne, R. H. Whiteley, R. E. Meads, J. Organomet. Chem. 1980, 193, 345-357.

[17] I. R. Butler, R. L. Davies, Synthesis. 1996, 1350-1354.

[18] a) P. Štěpnička, T. Baše, Inorg. Chem. Commun. 2001, 4, 682-687; b) P. Štěpnička, I. Císařová. Dalton Trans. 2013, 42, 3373-3389.

[19] a) J. M. Brunel, B. Faure, M. Maffei, Coord. Chem. Rev. 1998, 178-180, 665-698; b) H. Brisset, Y. Gourdel, P. Pellon, M. Le Corre, Tetrahedron Lett. 1993, 34, 4523-4526.

[20] M. Van Overschelde, E. Vervecken, S. G. Modha, S. Cogen, E. Van der Eycken, J. Van der Eycken, Tetrahedron 2009, 65, 6410-6415.

[21] Sheldrick, G.M., Crystal structure refinement with ShelXL, Acta Cryst., 2015, $C 71,3-8$.

[22] a) Sheldrick, G.M., ShelXT-Integrated space-group and crystal-structure determination, Acta Cryst., 2015, A71, 3-8; b) Sheldrick, G.M., ShelX, Acta Cryst., 2008, A64, 112-122. 
Lorsqu'un écrit scientifique issu d'une activité de recherche financée au moins pour moitié par des dotations de l'État, des collectivités territoriales ou des établissements publics, par des subventions d'agences de financement nationales ou par des fonds de l'Union européenne est publié dans un périodique paraissant au moins une fois par an, son auteur dispose, même après avoir accordé des droits exclusifs à un éditeur, du droit de mettre à disposition gratuitement dans un format ouvert, par voie numérique, sous réserve de l'accord des éventuels coauteurs, la version finale de son manuscrit acceptée pour publication, dès lors que l'éditeur met lui-même celle-ci gratuitement à disposition par voie numérique ou, à défaut, à l'expiration d'un délai courant à compter de la date de la première publication. Ce délai est au maximum de six mois pour une publication dans le domaine des sciences, de la technique et de la médecine et de douze mois dans celui des sciences humaines et sociales.

\section{Automated references}

[1]. a) P. Štěpnička in Ferrocenes: Ligands, Materials and Biomolecules, John Wiley \& Sons, Chichester, 2008; b) K. S. Gan, T. S. A. Hor, Ferrocenes: Homogeneous Catalysis, Organic

Synthesis, Material Science (Eds.: A. Togni, T. Hayashi), VCH Verlagsgesellschaft, Weinheim, Germany 1995; c) G. Jaouen, N. Metzler-Nolte (Eds.), Topics in Organometallic Chemistry, vol. 32: Medicinal Organometallic Chemistry, Springer, Berlin, 2010.

[2]. a) A. Fihri, P. Meunier, J.-C. Hierso, Coord. Chem. Rev. 2007, 251, 2017-2055; b) L.-X. Dai, X.-L. Hou (Eds.), Chiral Ferrocenes in Asymmetric Catalysis: Synthesis and Applications, Wiley-VCH, Weinheim, Germany, 2010. [3]. P. Štěpnička, Chem. Soc. Rev. 2012, 41, 4273-4305.

[4]. N. Dwadnia, J. Roger, N. Pirio, H. Cattey, J.-C. Hierso, Coord. Chem. Rev. 2018, 355, 74-100.

[5]. a) J. Podlaha, P. Štěpnička, J. Ludvik, I. Císařová, Organometallics 1996, 15, 543-550; b) P. Štěpnička, Eur. J. Inorg. Chem. 2005, 3787-3803; c) J. Schulz, P. Vosáhlo, F. Uhlik, I. Cisařová, P. Štěpnička, Organometallics 2017, 36, 1828-1841.

[6]. P. Štěpnička, Coord. Chem. Rev. 2017, 353, 223-246.

[7].J. Schulz, I. Císařová, P. Štěpnička, Organometallics. 2012, 31, 729-738; b) J. Schulz, F. Horký, I. Císařová, P. Štěpnička, Catalysts 2017, 7, art n 167.

[8]. a) O. Bárta, I. Cisařová, P. Štěpnička, J. Organomet. Chem. 2018, 855, 26-32; b) M. Zábranský, A. Machara, I. Císařová, P. Štěpnička, Eur. J. Inorg. Chem. 2017, 4850-4860; c) P. Štěpnička, M. Zábranský, I. Císařová, J. Organomet. Chem. 2017, 846, 193-200; d) M. Zábranský, I. Cisařová, P. Štěpnička, Eur. J. Inorg. Chem. 2017, 2557-2572; e) H. Charvátová, I. Císařová, P. Štěpnička, Eur. J. Inorg. Chem. 2017, 288-296; f) O. Bárta, I. Císařová, P. Štěpnička, Eur. J. Inorg. Chem. 2017, 489-495; g) K. Škoch, F. Uhlik, I. Cisařová, P. Štěpnička, Dalton. Trans. 2016, 45, 10655-10671; h) K. Škoch, I. Cisaroová, P. Štěennička, Chem. Eur. J. 2015, 21, 15998-16004.

[9]. a) N. Dwadnia, F. Allouch, N. Pirio, J. Roger, H. Cattey, S. Fournier, M.-J. Penouilh, C. H. Devillers, D. Lucas, D. Naoufal, R. Ben Salem, J.-C. Hierso, Organometallics 2013, 32, 5784-5797; b) F. Allouch, N. Dwadnia, N.V. Vologdin, Y.V. Svyaschenko, H. Cattey, M.-J. Penouilh, J. Roger, D. Naoufal, R. Ben Salem, N. Pirio, J.-C. Hierso, Organometallics. 2015, 34, 5015-5028; c) J.-C. Hierso, R. V. Smaliy, R. Amardeil, P. Meunier, Chem. Soc. Rev. 2007, 36, 1754-1769; d) J.-C. Hierso, M. Beaupérin, P. Meunier, Eur. J. Inorg. Chem. 2007, 3767-3780.

[10]. a) M. Beaupérin, R. Smaliy, H. Cattey, P. Meunier, J. Ou, P. H. Toy, J.-C. Hierso, Chem. Commun. 2014, 50, 9505-9508; b) M. Beaupérin, R. Smaliy, H. Cattey, P. Meunier, J. Ou, P. H. Toy, J.-C. Hierso, ChemPlusChem, 2015, 1, 119-129.

[11]. a) For gold-catalysed Suzuki cross-coupling, see: N. Dwadnia, J. Roger, N. Pirio, H. Cattey, R. Ben Salem, J.-C. Hierso, Chem. Asian J. 2017, 12, 459-464; For palladium-catalysed C-H functionalisation, see: b) D. Roy, S. Mom, M. Beaupérin, H. Doucet, J.-C. Hierso, Angew. Chem. Int. Ed. 2010, 49, 6650; Angew. Chem. 2010, 122, 6800; c) D. Roy, S. Mom, D. Lucas, H. Cattey, J.-C. Hierso, H. Doucet, Chem. Eur. J. 2011, 17, 6453; d) D. Roy, S. Mom, S. Royer, D. Lucas, J.-C. Hierso, H. Doucet, ACS Catal., 2012, 2, 1033; e) X. Wu, J. W. T. See, K. Xu, H. Hirao, J. Roger, J.-C. Hierso, J. Zhou, Angew. Chem. Int. Ed. 2014, 53, 13573; Angew. Chem. 2014, 126, 13791; f) For C-O etherification reactions, see: M. Platon, L. Cui, S. Mom, P. Richard, M. Saeys, J.-C. Hierso, Adv. Synth. Catal. 2011, 353, 3403; g) For C-S thioetherification reactions, see: M. Platon, N. Wijaya, V. Rampazzi, L. Cui, Y. Rousselin, M. Saeys, J.-C. Hierso, Chem. Eur. J. 2014, 20, 12584.

[12]. a) E. Lerayer, P. Renaut, J. Roger; N. Pirio, H. Cattey, C. H. Devillers, D. Lucas, J.-C. Hierso, Chem. Commun. 2017, 53, 6017-6020; b) G. Masson, P. Beyer, P. W. Cyr, A. J. Lough, I. Manners, Macromolecules 2006, 39, 3720-3730; c) M. Roemer, D. Heinrich, Y. K. Kang, Y. K. Chung, D. Lentz, Organometallics 2012, 31, 1500-1510.

[13]. E. Lerayer, P. Renaut, S. Brandès, H. Cattey, P. Fleurat-Lessard, G. Bouhadir, D. Bourisson, J.-C. Hierso, Inorg. Chem. 2017, 56, 1966-1973.

[14]. a) J. Roger, S. Royer, H. Cattey, A. Savateev, R. V. Smaliy, A. N. Kostyuk, J.-C. Hierso, Eur. J. Inorg. Chem. 2017, 330-339; b) V. Rampazzi, J. Roger, R. Amardeil, M.-J. Penouilh, P. Richard, P. Fleurat-Lessard, J.-C. Hierso, Inorg. Chem. 2016, 55, 10907-10921; c) R. A. Olofson, C. M. Dougherty, J. Am. Chem. Soc. 1973, 95, 977-584; d) W. McFarlane, D. S. Rycroft, J. Chem. Soc., Dalton Trans. 1973, 2162-2166; e) R. D. Kroshefsky, R. Weiss, J. G. Verkade, Inorg. Chem. 1979, 18, 469-472; f) D. W. Allen, B. F. Taylor, J. Chem. Soc., Dalton Trans. 1982, 51-54; g) M. N. Chevykalova, L. F. Manzhukova, N. V. Artemova, Y. N. Luzikov, I. E. Nifantev, E. E. Nifantev, Russ. Chem. Bull. 2003, 52, 78-84.

[15]. a) D. Seyferth, H. P. Withers Jr, J. Organomet. Chem. 1980, 185, C1-C5; b) D. Seyferth, H. P. Withers Jr, Organometallics 1982, 1, 1275-1282.

[16]. A. G. Osborne, R. H. Whiteley, R. E. Meads, J. Organomet. Chem. 1980, 193, 345-357.

[17]. I. R. Butler, R. L. Davies, Synthesis. 1996, 1350-1354.

[18]. a) P. Štěpnička, Inorg. Chem. Commun. 2001, 4, 682-687; b) P. Štěpnička, I. Císařová. Dalton Trans. 2013, 42, 3373-3389.

[19]. a) J. M. Brunel, B. Faure, M. Maffei, Coord. Chem. Rev. 1998, 178-180, 665-698; b) H. Brisset, Y. Gourdel, P. Pellon, M. Le Corre, Tetrahedron Lett. 1993, 34, 4523-4526.

[20]. M. Van Overschelde, E. Vervecken, S. G. Modha, S. Cogen, E. Van der Eycken, J. Van der Eycken, Tetrahedron, 2009, 65, 6410-6415.

[21]. Sheldrick, G.M., Crystal structure refinement with ShelXL, Acta Cryst., 2015, C71, 3-8.

[22]. a) Sheldrick, G.M., SheIXT-Integrated space-group and crystal-structure determination, Acta Cryst., 2015, A71, 3-8 ; b) Sheldrick, G.M., ShelX, Acta Cryst., 2008 , A64, $112-122$. 